\title{
.
}

\section{PSD3 downregulation confers protection against fatty liver disease}

\author{
Rosellina M. Mancina ${ }^{1}{ }^{1,31}$, Kavitha Sasidharan ${ }^{1}{ }^{1,31}$, Anna Lindblom ${ }^{2}{ }^{2}$, Ying Wei ${ }^{3}$, Ester Ciociola', \\ Oveis Jamialahmadi', Piero Pingitore', Anne-Christine Andréasson ${ }^{4}$, Giovanni Pellegrini ${ }^{5}$, \\ Guido Baselli ${ }^{6}{ }^{6}$, Ville Männistö7, Jussi Pihlajamäki ${ }^{8,9}$, Vesa Kärjä ${ }^{10}$, Stefania Grimaudo"11, \\ Ilaria Marini ${ }^{6}$, Marco Maggioni ${ }^{12}$, Barbara Becattini', Federica Tavaglione', Carly Dix ${ }^{13}$, Marie Castaldo ${ }^{14}$, \\ Stephanie Klein ${ }^{3}$, Mark Perelis ${ }^{3}$, Francois Pattou ${ }^{15,16}$, Dorothée Thuillier ${ }^{15}$, Violeta Raverdy ${ }^{15,16}$, \\ Paola Dongiovanni ${ }^{17}$, Anna Ludovica Fracanzani ${ }^{17,18}$, Felix Stickel ${ }^{19}$, Jochen Hampe ${ }^{20}$, \\ Stephan Buch ${ }^{20}{ }^{20}$, Panu K. Luukkonen ${ }^{21,22,23}$, Daniele Prati ${ }^{6}$, Hannele Yki-Järvinen ${ }^{21,22}$, Salvatore Petta", \\ Chao Xing ${ }^{24}$, Clemens Schafmayer ${ }^{25}$, Elmar Aigner ${ }^{26}$, Christian Datz ${ }^{27}$, Richard G. Lee ${ }^{3}$, Luca Valenti ${ }^{6}$, \\ Daniel Lindén $\oplus^{2,28,32 \bowtie ~ a n d ~ S t e f a n o ~ R o m e o ~} 1$ 1,29,30,32凶
}

Fatty liver disease (FLD) is a growing health issue with burdening unmet clinical needs. FLD has a genetic component but, despite the common variants already identified, there is still a missing heritability component. Using a candidate gene approach, we identify a locus (rs71519934) at the Pleckstrin and Sec7 domain-containing 3 (PSD3) gene resulting in a leucine to threonine substitution at position 186 of the protein (L186T) that reduces susceptibility to the entire spectrum of FLD in individuals at risk. PSD3 downregulation by short interfering RNA reduces intracellular lipid content in primary human hepatocytes cultured in two and three dimensions, and in human and rodent hepatoma cells. Consistent with this, Psd3 downregulation by antisense oligonucleotides in vivo protects against FLD in mice fed a non-alcoholic steatohepatitis-inducing diet. Thus, translating these results to humans, PSD3 downregulation might be a future therapeutic option for treating FLD.

atty liver disease (FLD) is a growing health issue and is already the leading cause of liver damage worldwide ${ }^{1}$. Liver fat accumulation is the hallmark of FLD, which covers a spectrum of conditions ranging from simple steatosis to liver inflammation and fibrosis ${ }^{2}$. Progression of FLD to advanced fibrosis may result in cirrhosis and hepatocellular carcinoma.

The worldwide prevalence of severe liver fibrosis related to FLD is expected to double within the next 10 years and become the leading

'Department of Molecular and Clinical Medicine, Institute of Medicine, Sahlgrenska Academy, Wallenberg Laboratory, University of Gothenburg, Gothenburg, Sweden. ${ }^{2}$ Bioscience Metabolism, Research and Early Development Cardiovascular, Renal and Metabolism (CVRM) BioPharmaceuticals R\&D, AstraZeneca, Gothenburg, Sweden. ${ }^{3}$ Ionis Pharmaceuticals, Carlsbad, CA, USA. ${ }^{4}$ Bioscience Cardiovascular, Research and Early Development Cardiovascular, Renal and Metabolism (CVRM) BioPharmaceuticals R\&D, AstraZeneca, Gothenburg, Sweden. ${ }^{5}$ Pathology, Clinical Pharmacology and Safety Sciences BioPharmaceuticals R\&D, AstraZeneca, Gothenburg, Sweden. ${ }^{6}$ Translational Medicine, Department of Transfusion Medicine and Hematology, Fondazione IRCCS Ca' Granda Ospedale Maggiore Policlinico and Department of Pathophysiology and Transplantation, Università degli Studi di Milano, Milan, Italy. ${ }^{7}$ Department of Medicine, University of Eastern Finland and Kuopio University Hospital, Kuopio, Finland. ${ }^{8}$ Institute of Public Health and Clinical Nutrition, University of Eastern Finland, Kuopio, Finland. ${ }^{9}$ Clinical Nutrition and Obesity Centre, Kuopio University Hospital, Kuopio, Finland. ${ }^{10}$ Department of Pathology, University of Eastern Finland and Kuopio University Hospital, Kuopio, Finland. "'Section of Gastroenterology and Hepatology, PROMISE, University of Palermo, Palermo, Italy. ${ }^{12}$ Department of Pathology, Fondazione Ca' Granda Ospedale Maggiore Policlinico, Milan, Italy. ${ }^{13}$ Antibody Discovery and Protein Engineering (ADPE), AstraZeneca, Cambridge, UK. ${ }^{14}$ Discovery Biology, Discovery Sciences R\&D, AstraZeneca, Gothenburg, Sweden. ${ }^{15}$ University of Lille, Inserm, Lille Pasteur Institute, CHU Lille, European Genomic Institute for Diabetes, U1190 Translational Research in Diabetes, Lille University, Lille, France. ${ }^{16} \mathrm{CHU}$ Lille, Department of General and Endocrine Surgery, Intergrated Center for Obesity, Lille, France. ${ }^{17} \mathrm{General}$ Medicine and Metabolic Diseases, Fondazione IRCCS Ca' Granda Ospedale Maggiore Policlinico, Milan, Italy. ${ }^{18}$ Department of Pathophysiology and Transplantation, Università degli Studi di Milano, Milan, Italy. ${ }^{19}$ Department of Gastroenterology and Hepatology, University Hospital of Zurich, Zurich, Switzerland. ${ }^{20}$ Medical Department 1, University Hospital Dresden, Technische Universitaät Dresden (TU Dresden), Dresden, Germany. ${ }^{21}$ Department of Medicine, University of Helsinki and Helsinki University Central Hosptial, Helsinki, Finland. ${ }^{22}$ Minerva Foundation Institute for Medical Research, Helsinki, Finland. ${ }^{23}$ Department of Internal Medicine, Yale University, New Haven, CT, USA. ${ }^{24}$ McDermott Center for Human Growth and Development University of Texas Southwestern Medical Center, Dallas, TX, USA. ${ }^{25}$ Department of General, Visceral, Vascular and Transplantation Surgery, University of Rostock, Rostock, Germany. ${ }^{26}$ First Department of Medicine, Paracelsus Medical University, Salzburg, Austria. ${ }^{27}$ Department of Internal Medicine, General Hospital Oberndorf, Teaching Hospital of the Paracelsus Medical University Salzburg, Oberndorf, Austria. ${ }^{28}$ Division of Endocrinology, Department of Neuroscience and Physiology, Sahlgrenska Academy, University of Gothenburg, Gothenburg, Sweden. ${ }^{29}$ Department of Cardiology, Sahlgrenska University Hospital, Gothenburg, Sweden. ${ }^{30} \mathrm{Clinical}$ Nutrition Unit, Department of Medical and Surgical Sciences, University Magna Graecia, Catanzaro, Italy. ${ }^{31 T h e s e}$ authors contributed equally: Rosellina M. Mancina, Kavitha Sasidharan ${ }^{32}$ These authors jointly supervised this work: Daniel Lindén, Stefano Romeo

凶e-mail: daniel.linden@astrazeneca.com; stefano.romeo@wlab.gu.se 


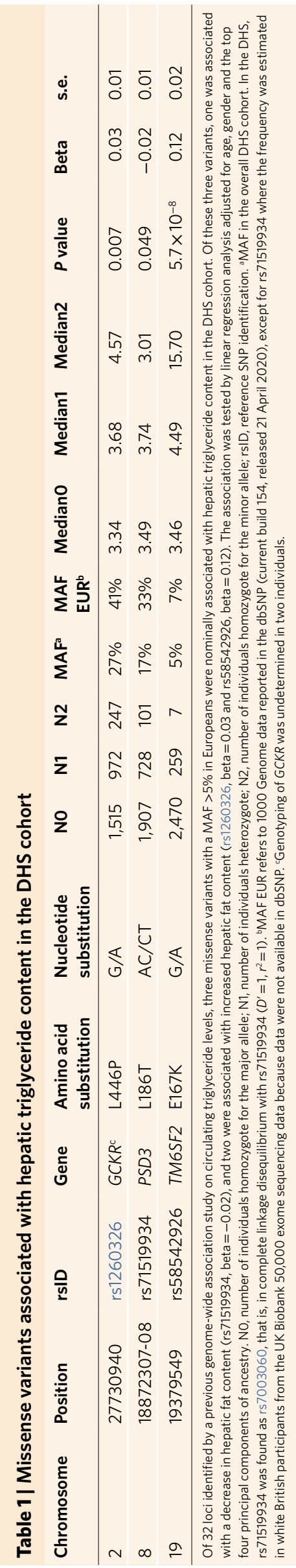

cause of liver failure and cancer, leading to a major threat to public health ${ }^{3}$. Despite promising results obtained with drugs targeting dysmetabolism, no drug has yet been approved for the treatment of FLD $^{4-6}$. Therefore, novel effective therapies and non-invasive biomarkers are urgently needed.

FLD is a highly heritable trait, with an estimated heritability of between $25 \%$ and $60 \%^{7-11}$. Genetic variations in Patatin-like phospholipase domain-containing protein 3 (PNPLA3) and other lipid droplet-related genes contribute to an increased risk of FLD onset and progression ${ }^{12-16}$. These genetic variations affect hepatic lipid handling in the liver by altering lipid secretion, lipid droplet remodelling or by increasing de novo lipogenesis, and result in liver inflammation and fibrosis ${ }^{17}$. Despite recent progress in the field, the common variants identified to date account for less than $6 \%$ of the disease variability ${ }^{17}$.

To identify additional loci affecting hepatic fat content, we used a candidate gene approach starting from gene variants previously identified through a genome-wide association study as determinants of fasting circulating triglycerides ${ }^{18}$. Fasting triglycerides are produced by the liver in the form of very low-density lipoproteins and are a proxy for hepatic fat content ${ }^{19-23}$.

In the discovery phase, we examined the association between variants in the candidate genes and liver fat content in the Dallas Heart Study (DHS) cohort $(n=2,736)$. Next, we validated our results in: (1) the Liver Biopsy Cohort (LBC; $n=1,951)$ consisting of individuals at risk for non-alcoholic fatty liver disease (NAFLD) from northern and southern Europe; (2) participants from the United Kingdom (UK) Biobank cohort for whom liver fat measurements were available $(n=10,970)$; and (3) an independent cohort of individuals with obesity at risk for liver disease from central Europe with liver biopsy available $(n=674)$. To better understand the molecular mechanisms underlying the genetic association, we performed studies in mice, in human primary hepatocytes and in human and rodent hepatoma cells.

We identified a locus, previously not linked to liver fat content in the Pleckstrin and Sec7 domain-containing 3 (PSD3) gene protecting against liver fat accumulation, inflammation and fibrosis. Downregulation of this gene conferred protection against the entire spectrum of FLD in mice, and against lipid accumulation in primary human hepatocytes and in human and rodent hepatoma cells.

\section{Results}

PSD3 sequence variation reduces FLD susceptibility in humans. The study design is shown in Supplementary Fig. 1. To identify previously unknown genetic loci affecting liver fat content, we used a candidate gene approach in the DHS, a population-based sample study with measurement of liver fat content by proton magnetic spectroscopy in 2,736 participants. Specifically, we selected all the tag single-nucleotide polymorphisms (SNPs) identified by a previous genome-wide study on circulating triglyceride levels ${ }^{18}$ (Supplementary Table 1). All missense and nonsense variants in the genes identified by the tag SNPs were then tested for association with liver fat content in the $\mathrm{DHS}^{14}$ using linear regression analysis under an additive genetic model adjusted for age, gender and the top four principal components of ancestry. For tag SNPs that were intergenic, missense and nonsense variants in both genes flanking the tag SNP were examined.

We identified three missense variants in three genes present in Europeans (minor allele frequency (MAF) $>5 \%$ in Europeans from the 1000 Genome Project, Database of Single Nucleotide Polymorphisms (dbSNP), current build 154, released 21 April 2020) that were nominally associated $(P<0.050)$ with liver fat content in the DHS (Table 1).

Among these, we found two variants robustly associated with FLD and circulating triglycerides, namely the Glucokinase regulator $(G C K R)$ rs1260326 $(P=0.007)$ and Transmembrane 6 Superfamily 
Table 2 | PSD3 rs71519934 minor allele associated with lower prevalence of liver disease in the LBC ( $N$ histological data =1,951)

\begin{tabular}{|c|c|c|c|c|c|c|c|}
\hline & $186 \mathrm{~L}$ & L186T & $186 \mathrm{~T}$ & $P$ value & OR & $\mathrm{Cl}$ & \\
\hline$N$ & 917 & 796 & 238 & & & & \\
\hline Steatosis presence, $n(\%)$ & $778(85)$ & $603(76)$ & $170(71)$ & $5.9 \times 10^{-6}$ & 0.67 & 0.57 & 0.80 \\
\hline Fibrosis presence, $n(\%)$ & $536(58)$ & $417(52)$ & $121(51)$ & 0.006 & 0.82 & 0.72 & 0.94 \\
\hline Inflammation presence, $n(\%)$ & $596(65)$ & $420(53)$ & $118(50)$ & $9.9 \times 10^{-7}$ & 0.70 & 0.61 & 0.81 \\
\hline Ballooning presence, $n(\%)^{a}$ & $338(39)$ & $246(34)$ & $68(31)$ & 0.002 & 0.79 & 0.68 & 0.92 \\
\hline
\end{tabular}

The association was tested by binary logistic regression analysis under an additive genetic model adjusted by age, gender, BMI, centre of recruitment and number of PNPLA3 mutant alleles. Presence of steatosis, fibrosis, inflammation or ballooning was defined as the relative degree $>0$. $\mathrm{Cl}$, confidence interval; OR, odds ratio. ${ }^{\mathrm{a}} \mathrm{Data}$ available for $n=1,805$.

Member 2 (TM6SF2) rs58542926 $\left(P=5.7 \times 10^{-8}\right)^{15,24}$. Moreover, we found a variant in the PSD3 gene, rs71519934, associated with lower liver fat content $(P=0.049)$. The DHS consists of three ethnic groups, namely European American, African American and Hispanic American. We next examined differences in clinical and anthropometric parameters, lipoprotein levels and liver fat content stratified by PSD3 rs71519934 genotype and by these ethnic groups (Supplementary Table 2). In the entire DHS there was no difference in these selected traits among the three genotypes, except for liver fat content. However, when stratified by ethnicity, there was a reduction in circulating total cholesterol levels for the PSD3 rs71519934 genotype in European Americans.

The PSD3 rs71519934, in the dbSNP, is annotated as a threonine to leucine amino acid substitution at position 186 of the PSD3 protein (dbSNP, current build 154, released 21 April 2020; https://www. ncbi.nlm.nih.gov/snp/rs71519934). However, exome sequencing of participants from the UK Biobank revealed that the 186 threonine is the minor allele in Europeans (Supplementary Fig. 2).

To test whether the PSD3 rs71519934 is associated with protection against the entire spectrum of FLD, we examined this variant in the LBC, comprising $n=1,951$ Europeans at high risk for FLD with liver biopsy available (Supplementary Table 3). In the LBC, the PSD3 rs71519934 minor allele (186T) was associated with lower prevalence of liver steatosis $\left(P=5.9 \times 10^{-6}\right)$, fibrosis $(P=0.006)$, inflammation $\left(P=9.9 \times 10^{-7}\right)$ and ballooning $(P=0.002)$ (Table 2$)$ using binary logistic regression analysis under an additive genetic model adjusted for age, gender, body mass index (BMI), centre of recruitment and the PNPLA3 rs738409.

In addition, carriers of the $186 \mathrm{~T}$ minor allele were protected against more severe liver steatosis $\left(P=3.3 \times 10^{-7}\right)$, inflammation $\left(P=1.6 \times 10^{-7}\right)$, ballooning $(P=0.001)$ and fibrosis $(P=0.001)$ (Fig. 1a-d). These results were virtually identical when further adjusted for other genetic (TM6SF2 rs58542926 (E167K), MBOAT7 rs641738 and GCKR rs1260326 (L446P)) and environmental (presence of diabetes) variables influencing FLD (Supplementary Table 4). Moreover, consistent with European Americans in the DHS, carriers of the PSD3 186T minor allele had lower circulating total and low-density lipoprotein (LDL) cholesterol levels $\left(P=1.4 \times 10^{-6}\right.$ and $P=0.001$, respectively; Supplementary Table 5). Next, we tested for an interaction between the PSD3 and PNPLA3 variants in the LBC but found no interaction between these two genetic variants and liver disease (Supplementary Fig. 3).

To seek independent replication of the association between the PSD3 variant and liver fat content, we examined white British participants $(n=10,970)$ from the UK Biobank with liver fat content measured as magnetic resonance imaging (MRI)-derived proton density fat fraction (PDFF). In this population-based study, genetic data on the rs71519934 dinucleotide substitution were not available, therefore we used the rs7003060 which is in complete linkage disequilibrium $\left(D^{\prime}=1, r^{2}=1\right)$ with the rs71519934 in Europeans. We did not find any association between the PSD3 minor allele and lower liver fat content in the UK Biobank (Table 3).
However, because genetic variations affecting FLD have a robust gene-environment interaction, with BMI amplifying their effect ${ }^{25}$, we examined the differences in liver fat content among the PSD3 rs7003060 genotype after stratification for severity of overweight/ obesity as measured by BMI. Severely obese (BMI > 35) carriers of the PSD3 minor allele had a lower liver fat content (beta $=-0.175$, $P=0.02$; Supplementary Fig. 4 and Table 3).

Considering these results, we further tested the association between the PSD 3 variant and the protection against liver disease in an independent replication cohort of obese $\left(B M I \geq 30 \mathrm{~kg} \mathrm{~m}^{-2}\right)$ central European participants at risk for liver disease ${ }^{26}$. More specifically, we examined 674 adult individuals (mean age $45 \pm 12$ years), with a mean BMI of $46 \pm 10 \mathrm{~kg} \mathrm{~m}^{-2}$ from three central European centres (Supplementary Table 3) with liver biopsies available. We found that the PSD3 minor allele was associated with a lower prevalence of liver steatosis $(P=0.024)$, fibrosis $(P=0.049)$ and ballooning $(P=0.047)$, and with less severe fibrosis and ballooning $(P=0.040$ and $P=0.048$, respectively; Table 4$)$. No differences in clinical or metabolic traits were detected in this cohort stratified by PSD3 genotype (Supplementary Table 5). Finally, we performed a meta-analysis of the LBC and the replication cohort from central Europe. With both fixed and random effect models, the PSD3 genetic association was stronger for all the traits examined, except for the presence and severity of inflammation where the association was attenuated using a random effect model (Supplementary Table 6).

PSD3 hepatic expression is increased in individuals with FLD. The PSD3 protein has 18 annotated isoforms (Ensembl release 75). To determine which isoform is the most abundant in human liver, we examined the transcriptome of liver biopsies from a subset of individuals $(n=77)$ from the $\mathrm{LBC}^{27}$. We found that isoform-a (annotated as NP_056125 in NCBI and as 001 ENST00000327040 in Ensembl) had the highest expression level in the liver (Fig. 2a). Interestingly, the total hepatic PSD3 messenger RNA levels were higher in livers with FLD than in those without (Fig. 1e), whereas no differences in the N-acetyltransferase 2 (NAT2) mRNA level were observed (Fig. 1f), When we stratified individuals based on the PSD3 genotype, we found no difference in the mRNA expression level of either PSD3 or NAT2 (Fig. 2b,c).

PSD3 186T protects against intracellular lipid accumulation. To understand the mechanism(s) underlying the association between the rs71519934 minor allele and lower liver fat content, we examined primary human hepatocytes from donors carrying the $186 \mathrm{~L}$ or $186 \mathrm{~T}$ amino acid change in homozygosity. Consistent with the genetic association, human primary hepatocytes homozygous for the $186 \mathrm{~T}$ allele cultured in two dimensions (2D) had a lower neutral lipid fat content $(P=0.007)$ as measured by Oil Red O (ORO) staining compared with homozygous 186L hepatocytes (Fig. 3a). To examine PSD3 levels in the two different genotypes, we generated an antibody specific for human PSD3 (custom antibody generation 
a

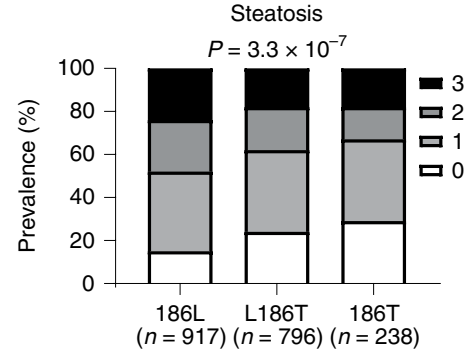

C
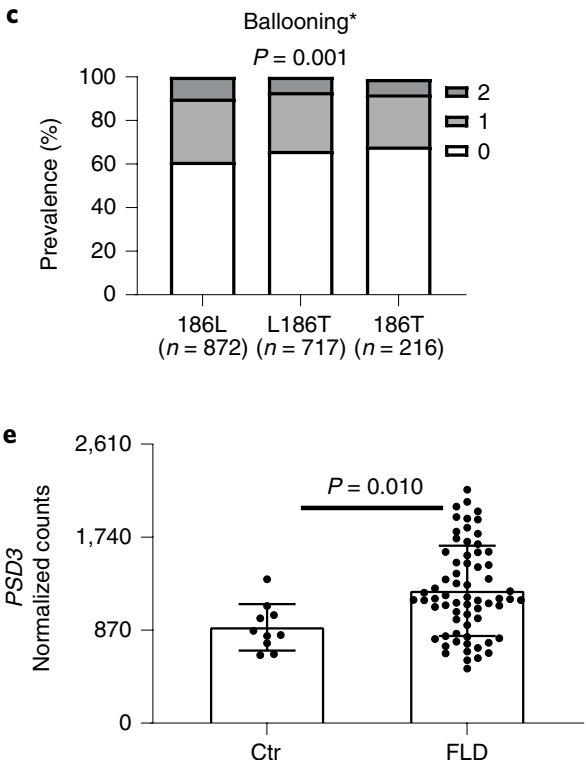

b

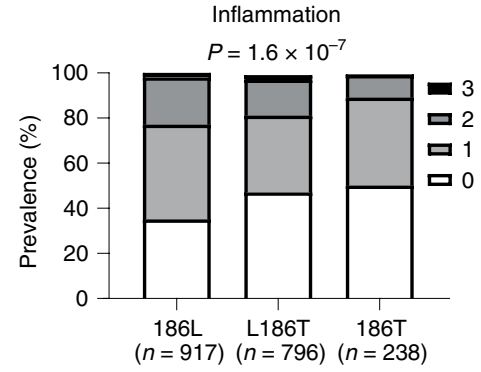

d

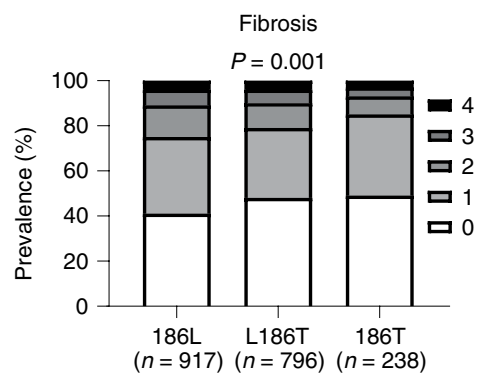

f

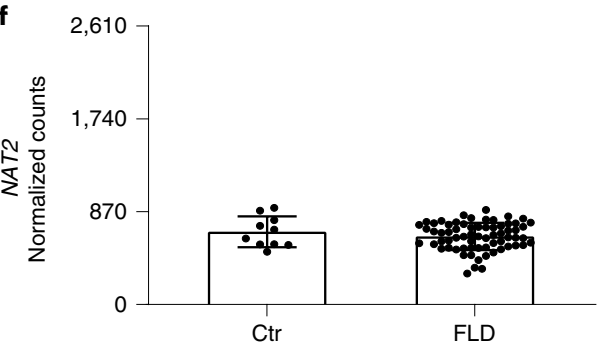

Fig. 1 | PSD3 minor allele protected against enhanced severity of histological liver damage in the LBC and PSD3 gene expression was higher in livers with FLD. a-d, Histological liver damage stratified by PSD3 genotype. Bars show the prevalence expressed as percentage of the degree of the specified disease. Colour shading from white to black indicates increased disease severity. Histological damage was evaluated according to the different components of the NAS and hepatic fibrosis stage. Carriers of the PSD3 rs71519934 186T minor allele had less severe liver disease with lower degrees of steatosis (a), inflammation (b), ballooning (c) and fibrosis (d). The association was tested under an additive genetic model by an ordinal regression analysis adjusted for age, gender, BMI, recruitment centre and number of PNPLA3 I148M mutant alleles. Results were virtually the same after further adjustment for the other main genetic risk factors (TM6SF2 rs58542926 (E167K), MBOAT7 rs641738 and GCKR rs1260326 (L446P)) and environmental (presence of diabetes) variables influencing FLD (Supplementary Table 4). All the reported $P$ values are two-sided with no adjustment for multiple testing. ${ }^{*}$ Data available for ballooning, $n=1,805$. e, Total PSD3 mRNA expression stratified by healthy and FLD livers. Liver PSD3 expression levels were higher in FLD subjects compared with healthy controls. f, Total NAT2 mRNA expression stratified by healthy and FLD livers. There was no difference in the NAT2 expression level based on the presence of FLD. e,f, Data are presented as mean and s.d. Two-sided $P$ value was calculated by Mann-Whitney non-parametric test. Ctr, healthy control livers $(n=10)$; FLD, livers with fatty liver disease $(n=67)$.

Table 3 | Association of PDFF with PSD3 rs7003060 overall and within four BMI strata in white British participants from the UK Biobank

rs7003060

\begin{tabular}{lccccccccc}
\hline & \multicolumn{2}{c}{ GG } & \multicolumn{1}{c}{ GT } & \multicolumn{2}{c}{ TT } & & \\
\hline & $\mathbf{N}$ & Median (i.q.r.) & $\boldsymbol{N}$ & Median (i.q.r.) & $\boldsymbol{N}$ & Median (i.q.r.) & $\boldsymbol{P}$ value & Beta & Cl \\
\hline Overall & 5,243 & $2.92(2.78)$ & 4,668 & $2.84(2.71)$ & 1,059 & $2.86(2.67)$ & 0.49 & -0.00892 & $-0.034,0.016$ \\
$\mathrm{BMI}<25$ & 2,137 & $2.18(1.5)$ & 1,907 & $2.19(1.40)$ & 442 & $2.22(1.36)$ & 0.77 & -0.00636 & $-0.048,0.035$ \\
$25 \leq \mathrm{BMI}<30$ & 2,294 & $3.32(2.94)$ & 2,019 & $3.29(3.10)$ & 433 & $3.31(2.85)$ & 0.66 & 0.00975 & $-0.033,0.052$ \\
$30 \leq \mathrm{BMI}<35$ & 651 & $4.86(6.03)$ & 568 & $4.25(4.87)$ & 143 & $4.72(6.67)$ & 0.18 & -0.0534 & $-0.13,0.025$ \\
$\mathrm{BMI} \geq 35$ & 156 & $6.37(8.8)$ & 165 & $5.14(8.04)$ & 39 & $5.60(7.47)$ & 0.02 & -0.175 & $-0.33,-0.022$ \\
\hline
\end{tabular}

Analysis was performed using a linear regression adjusted for age, sex, BMI, the first ten principal components of ancestry and array type.

detailed in Methods). We incubated cells with different amount of oleic acid $(\mathrm{OA})(0,10$ and $25 \mu \mathrm{M})$ and examined protein levels between the two genotypes in primary hepatocytes. Overall, the amount of PSD3 was elevated with increasing concentration of OA (Fig. 3b). However, for each OA concentration, PSD3 protein expression was lower in cells homozygous for the $186 \mathrm{~T}$ allele. To 
Table 4 | PSD3 rs71519934 minor allele was associated with a lower prevalence and lower severity of liver disease in the central Europeans independent replication cohort $(N$ histological data $=674)$

\begin{tabular}{|c|c|c|c|c|c|c|c|}
\hline & $186 \mathrm{~L}$ & L186T & $186 \mathrm{~T}$ & $P$ value & OR & $\mathrm{Cl}$ & \\
\hline$N$ & 330 & 282 & 62 & & & & \\
\hline \multicolumn{8}{|c|}{ Disease presence } \\
\hline Fibrosis & $132(40)$ & $103(36)$ & - & 0.049 & 0.77 & 0.59 & 1.00 \\
\hline Inflammation & $107(32)$ & $86(30)$ & $19(31)$ & 0.524 & 0.92 & 0.70 & 1.20 \\
\hline Ballooning & $106(32)$ & $72(25)$ & $18(29)$ & 0.047 & 0.75 & 0.56 & 1.00 \\
\hline Steatosis & & & & 0.158 & 0.85 & 0.69 & 1.06 \\
\hline Fibrosis & & & & 0.040 & 0.77 & 0.60 & 0.99 \\
\hline Inflammation & & & & 0.355 & 0.88 & 0.68 & 1.15 \\
\hline Ballooning & & & & 0.048 & 0.75 & 0.57 & 1.00 \\
\hline
\end{tabular}

The association was tested by binary logistic (disease presence) or ordinal regression (disease severity) analysis under an additive genetic model adjusted by age, gender, BMI, centre of recruitment and number of PNPLA3 mutant alleles. The OR for ordinal regression was calculated as exponentials of the coefficient estimate and its $\mathrm{Cl}$. Presence of steatosis, fibrosis, inflammation or ballooning was defined as the relative degree $>0$.
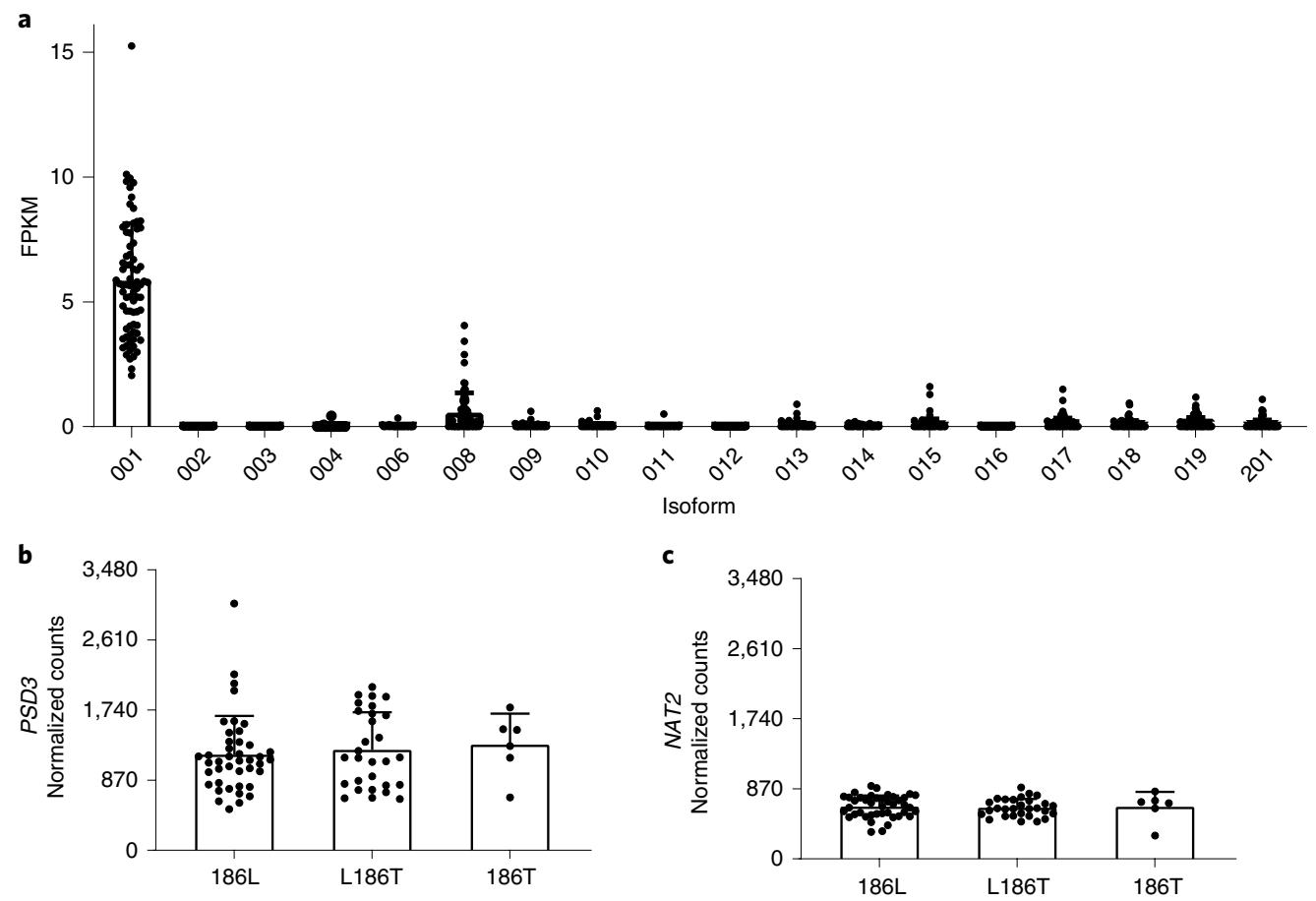

Fig. 2 | PSD3 mRNA isoform expression in human liver and total PSD3 mRNA expression stratified by rs71519934 genotype. a, Expression level of the different PSD3 mRNA isoforms in human liver tissue. To assess which isoform is the most abundant, the transcriptome from liver biopsies of a subset of 77 individuals from the Milan subgroup of the LBC was examined. Isoform 001 (identified as ENST00000327040 by Ensembl or as NP_056125 (isoform-a) by $\mathrm{NCBI}, 1,047$ amino acids) had the highest expression, followed by isoform 008 (identified as ENST00000521841 by Ensembl, non-coding). Data are presented as mean and s.d. b. Total PSD3 mRNA expression stratified by rs 71519934 genotype. There were no differences in PSD3 mRNA expression levels when stratified by genotype. c, Total NAT2 mRNA expression levels did not differ among PSD3 rs71519934 genotypes. b,c, Data are presented as mean and s.d. The two-sided $P$ value was calculated by unadjusted linear regression. Values were log-transformed before entering the model. FPKM, fragments per kilobase of exon model per million reads mapped; 186L, homozygotes for the L allele $(n=42)$; L186T, heterozygotes $(n=29)$; 186T, homozygotes for the T allele $(n=6)$.

understand the mechanism behind this association, we examined differentially expressed genes involved in lipid homoeostasis using RNA sequencing (RNA-seq). We found a robust reduction in genes involved in triglyceride synthesis and secretion and cholesterol biosynthesis, whereas the expression of $P G C-1 \alpha$ involved in mitochondrial biogenesis was increased (Fig. 3c).
PSD3 downregulation reduces intracellular lipid accumulation. Because PSD3 expression was elevated in livers from individuals with FLD, we tested the hypothesis that PSD3 downregulation would result in a reduction in intracellular fat content by using siRNA in human primary hepatocytes from donors homozygous for either the 186L or 186T allele. Downregulation of PSD3 reduced 


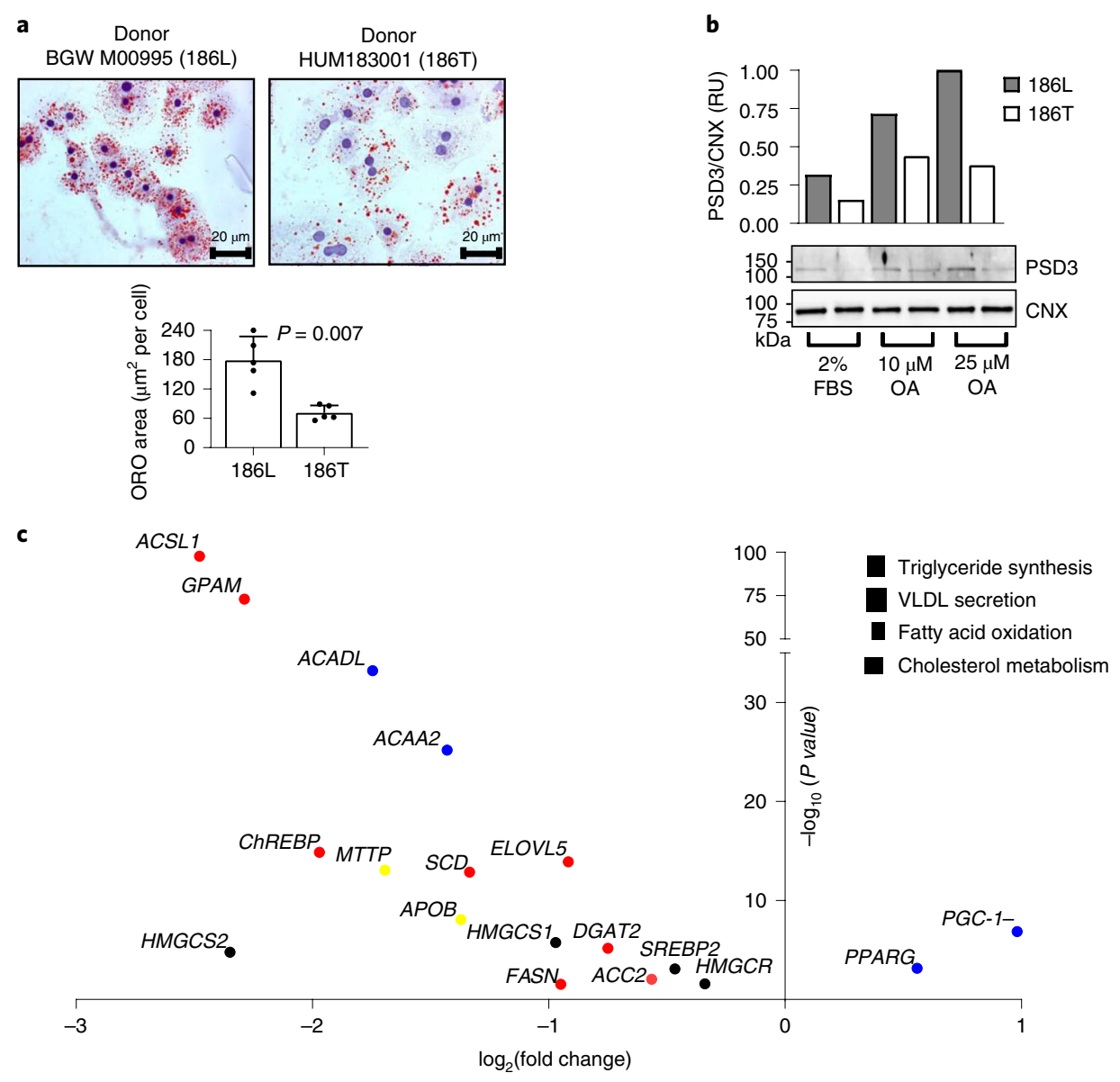

Fig. 3 | Primary human hepatocytes from a donor homozygous for the 186T allele had lower PSD3 protein and intracellular lipid levels compared with homozygous $186 \mathrm{~L}$ hepatocytes. Primary human hepatocytes from donors homozygous for 186L or 186T, were cultured in 2D. a, Intracellular neutral fat content visualized by ORO staining and quantified by Biopix iQ software v.2.3.1. Data are presented as mean and s.d. of the reported independent experiments $(n=5)$. Two-sided $P$ value was calculated by Mann-Whitney non-parametric test. b, Cells were cultured in serum-free regular medium supplemented with $2 \% \mathrm{FBS}, 10 \mu \mathrm{M}$ OA or $25 \mu \mathrm{M}$ OA for $48 \mathrm{~h}$. Immunoblotting was performed with total cell lysates to detect PSD3 (NCBI: NP_056125, 1,047 amino acids) using a custom antibody. The bar graph shows the relative PSD3 amount calculated as PSD3/calnexin $(C N X)$. c, Key genes involved in lipid metabolism that were differentially expressed between donors homozygous for 186T versus $186 \mathrm{~L}$ obtained with RNA-seq. Data are presented as $\log _{2}$ (fold change) in expression and $-\log _{10}$ ( $P$ value) adjusted using the Benjamini and Hochberg's approach for controlling the FDR. RU, relative units; VLDL, very low-density lipoprotein. Red Triglyceride synthesis; yellow VLDL secretion; blue Fatty acid oxidation; black cholesterol metabolism.

the intracellular neutral lipid content in hepatocytes carrying either allele when cultured in 2D (Fig. 4a,b). However, PSD3 silencing reduced intracellular lipid levels only in primary hepatocytes carrying the $186 \mathrm{~L}$ allele when cultured in a three-dimensional (3D) spheroid model (Fig. 4c,d).

PSD3 downregulation reduces triglyceride synthesis and ARF6 activation. To understand the molecular mechanism(s) underlying the genetic association, we examined immortalized hepatocytes from rat (McArdle, McA-RH7777) homozygous for 180T, which corresponds to $186 \mathrm{~T}$ in human PSD3. In these cells, Psd3 downregulation resulted in a reduction in the intracellular neutral lipid content as measured by ORO staining. Additionally, Psd3 downregulation in McA-RH7777 cells resulted in lower triglyceride production, measured as de novo triglyceride synthesis, and as mRNA expression of genes involved in triglyceride synthesis. Moreover, very low-density lipoprotein secretion, measured as apolipoprotein B (Apo B) secretion, was also lower in cells transfected with Psd3 siRNA compared with scramble (SCR) control siRNA. No differences were detected in intracellular lipid utilization, measured as beta oxidation (Supplementary Fig. 5). To confirm our data, we measured the intracellular lipid accumulation and triglyceride synthesis using radiolabelled tracers in human hepatoma cells (Huh7 cells) homozygous for the PSD3 186L allele, and obtained virtually identical results as McA-RH7777 cells (Supplementary Fig. 6). Thus, downregulation of endogenously expressed Psd 3 threonine or PSD3 leucine resulted in decreased intracellular lipid levels. PSD3 is a guanine nucleotide exchange factor activating ADP-ribosylation factor 6 (ARF6). To test whether PSD3 downregulation results in changes in ARF6 activation, PSD3 was downregulated in Huh7 cells and levels of activated ARF6 were measured using a GGA3 protein-binding domain (PBD) pull-down assay. PSD3 downregulation resulted in lower levels of activated ARF6 (ARF-GTP) (Fig. 5).

In immunohistochemistry analyses, PSD3 was found to be present at a low level in hepatocytes and there was no apparent difference in the levels between L186T heterozygous and 186L homozygous donors (Supplementary Fig. 7). Otherwise, sinusoidal endothelial cells and biliary ducts showed moderate positivity. Interestingly however, the sample from one heterozygous patient characterized by the lowest grade of steatosis also showed the weakest staining 
a

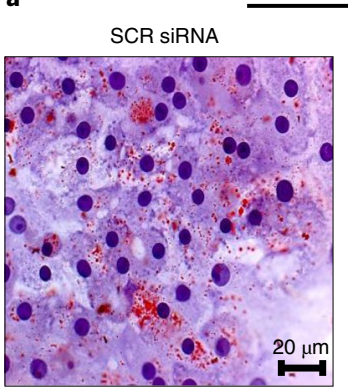

PSD3 siRNA
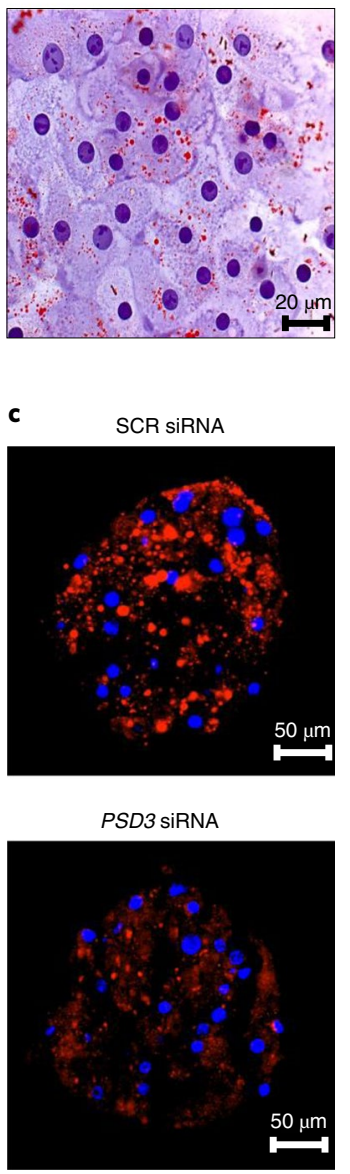
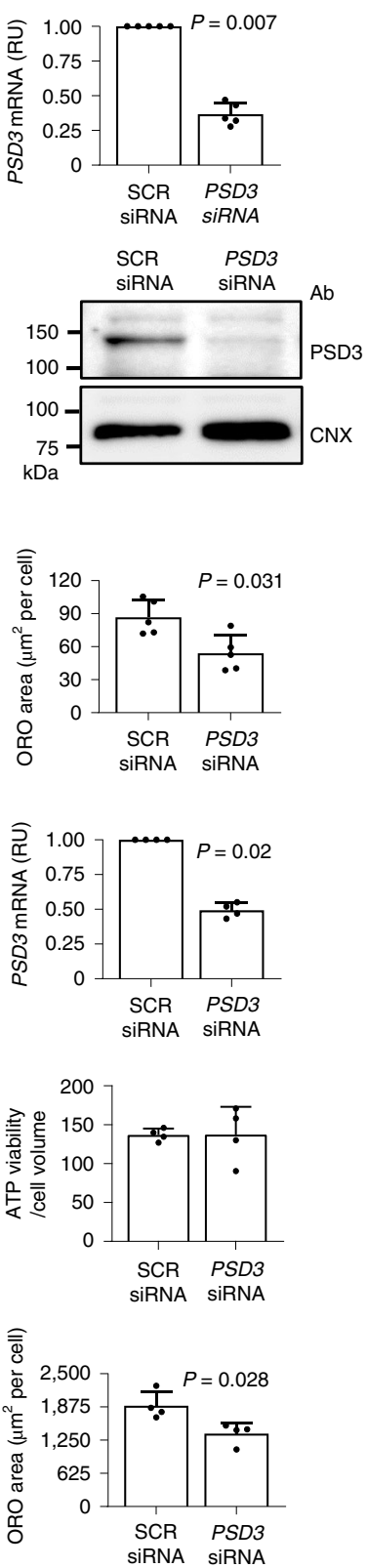

b
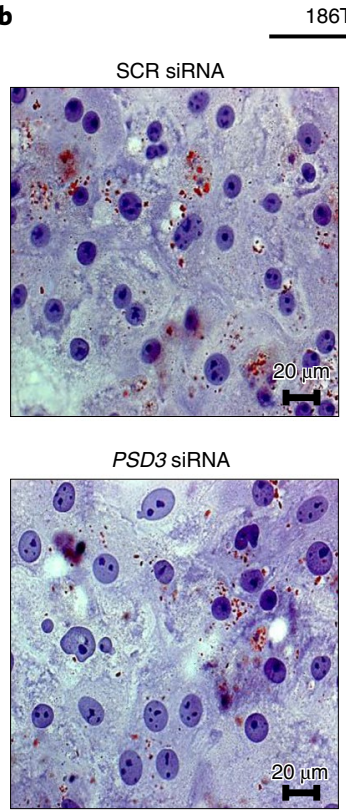

d
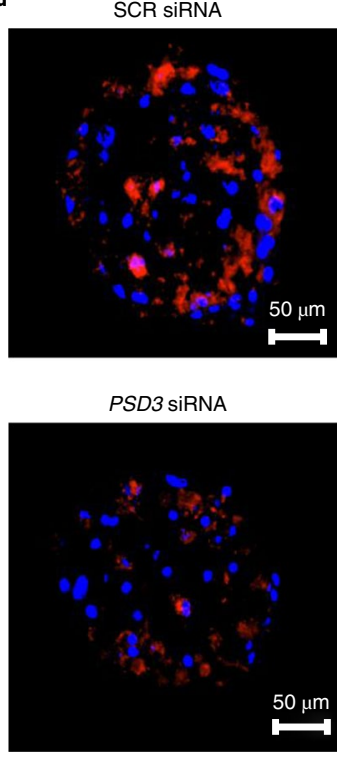

$86 \mathrm{~T}$
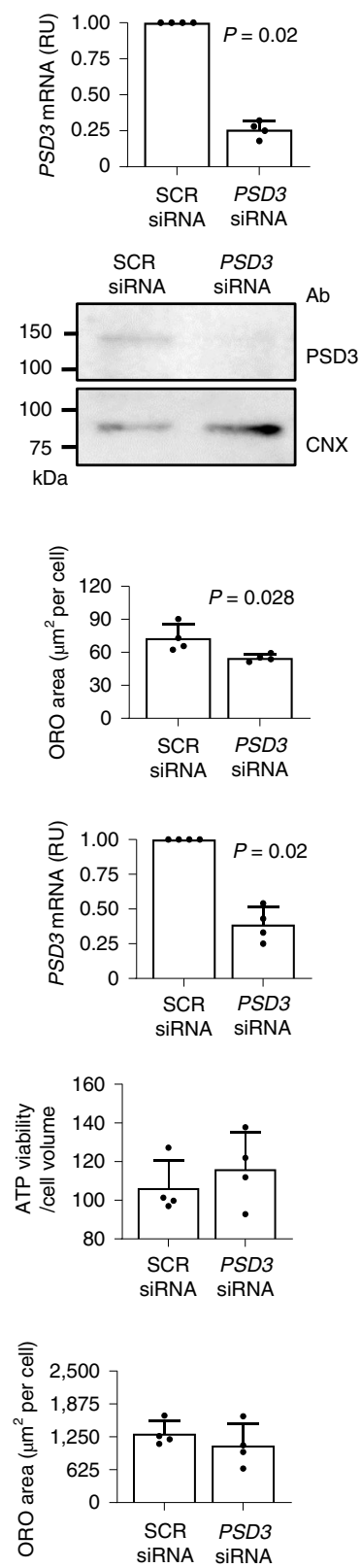

Fig. 4 | PSD3 downregulation lowered intracellular neutral fat content in primary human hepatocytes cultured in 2D and 3D spheroids. Downregulation of endogenous PSD3 expression using siRNA in primary human hepatocytes cultured in 2D and 3D. For 2D culture, after attachment of cells in collagen-coated plates, cells were incubated with regular growth medium supplemented with $10 \mu \mathrm{M} O \mathrm{O}$ and transfected with negative control SCR siRNA or PSD3 siRNA for 48 h. a,b, Intracellular neutral fat content was visualized by ORO staining and quantified by Biopix iQ software v.2.3.1 in primary human hepatocytes carrying $(\mathbf{a})$ the $186 \mathrm{~L}$ allele $(n=5)$ or $(\mathbf{b})$ the $186 \mathrm{~T}$ allele $(n=4)$. Average PSD3 downregulation efficiency was $~ 80 \%$ as evaluated by quantitative retro transcription PCR analysed by the $2^{-\Delta \Delta \mathrm{Ct}}$ method and western blotting for both donor types. For 3D culture of primary human hepatocytes, spheroids were generated by seeding 2,000 cells per well in a 96-well round-bottom flask, along with transfection mix in $100 \mu$ l of medium. For the generation of $186 \mathrm{~T}$ allele spheroids, $5 \mathrm{nM}$ of FMK-Z-VAD was added to support spheroid formation. After $24 \mathrm{~h}$, additional growth medium was added to give a total volume of $200 \mu \mathrm{l}$ per well. Fifty per cent of the total media was replenished with fresh media every $48 \mathrm{~h}$. After 7 days of formation, spheroids were collected and $8-\mu \mathrm{M}$ sections were subjected to ORO staining to visualize intracellular neutral fat content. c,d, Nuclei were stained with 4,6-diamidino-2-phenylindole and ORO staining was quantified by Image J, normalized to number of nuclei of primary human hepatocyte spheroids carrying $(\mathbf{c})$ the $186 \mathrm{~L}$ allele $(n=4)$ and $(\mathbf{d})$ the $186 \mathrm{~T}$ allele $(n=4)$. Average gene knockdown efficiency was $\sim 50-60 \%$ as evaluated by quantitative retro transcription PCR analysed by the $2^{-\Delta \Delta C t}$ method for both donor types. Cellular ATP levels (marker of viability) remained stable between the negative control SCR and PSD3 siRNA groups. Data are shown as mean \pm s.d. of the reported independent experiments. Two-sided $P$ values were calculated by Mann-Whitney non-parametric test comparing SCR siRNA versus PSD3 siRNA. RU, relative units; CNX, calnexin.

intensity for PSD3. Regarding ARF6, hepatocytes showed multifocally moderate cytoplasmic and membrane positivity. The staining for activated ARF6, especially in membranes, appeared more pro- nounced in samples homozygous for 186L (Supplementary Fig. 7). However, immunohistochemistry does not allow for the quantification of PSD3 or activated ARF6. 

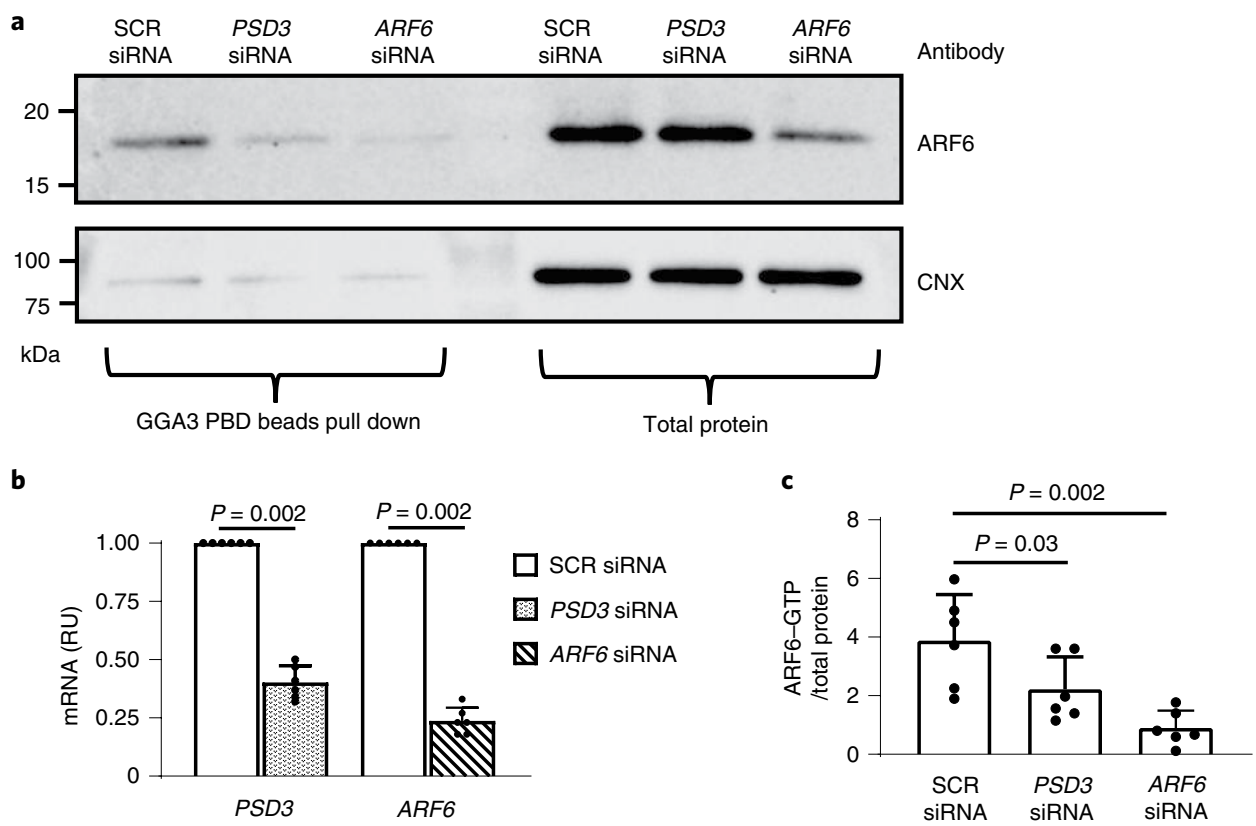

Fig. 5 | Downregulation of PSD3 resulted in partial loss of ARF6 activation. Human hepatoma Huh7 cells were transiently transfected with negative control SCR siRNA, PSD3 siRNA or ARF6 siRNA. Forty-eight hours after transfection, the cell lysates were incubated with GGA3 PBD agarose beads that selectively isolate and pull down endogenous active ARF6 (ARF6-GTP). a, After precipitation, active ARF6-GTP was detected by immunoblotting using an anti-ARF6 antibody provided in the kit. Cells transfected with ARF6 siRNA were used as a control. The experiment was performed independently six times with similar results. Representative blot presented. $\mathbf{b}$, Knockdown efficiency with $\sim 60 \%$ reduction for PSD3 and $\sim 75 \%$ for ARF6 as evaluated by quantitative retro transcription PCR analysed by the $2^{-\Delta \Delta \mathrm{Ct}}$ method $(n=6)$. c, Relative ARF6-GTP (active) calculated as GTP-ARF6/calnexin (CNX) $(n=6)$. Data are shown as mean \pm s.d. of the reported independent experiments. Two-sided $P$ values calculated by Mann-Whitney non-parametric test. $R U$, relative units.

Psd3 downregulation protects against FLD in mice. Next, we downregulated liver $P s d 3$ in vivo by administrating triantennary $\mathrm{N}$-acetylgalactosamine (GalNAc)-conjugated Gen 2.5 antisense oligonucleotides (ASOs) in C57BL/6 mice. Mice were fed a non-alcoholic steatohepatitis (NASH)-inducing diet for a total of 50 weeks, and during the last 16 weeks, groups of mice were treated with Psd3 ASO, control ASO or saline. Psd3 ASO treatment decreased the liver Psd3 mRNA expression levels by $98 \%$ (Fig. 6a) and reduced the liver weight, total liver triglyceride content and plasma alanine transaminase (ALT) levels (Fig. 6b-d). Psd3 ASO treatment did not affect the body weight of the mice (Fig. 7a).

Moreover, Psd3 ASO treatment reduced the total liver free cholesterol, liver cholesteryl ester, plasma aspartate transaminase (AST), total and LDL cholesterol levels but did not change the plasma triglyceride or high-density lipoprotein (HDL) cholesterol levels (Fig. 7b-h). Histologically, Psd3 ASO treatment reduced the liver collagen 1a1 (Colla1) protein and liver lipid droplet levels (Figs. $6 \mathrm{e}, \mathrm{f}$ and 8 ). Next, to examine the severity of liver disease using the semiquantitative scoring developed by Kleiner et $\mathrm{al}^{28}$, we performed ordinal regression analyses and found that Psd3 ASO treatment reduced the severity of steatosis and lobular inflammation scores, and the NAFLD activity score (NAS), although the liver fibrosis score did not change significantly (Fig. $6 \mathrm{~g}-\mathrm{j}$ ). To gain insights into the mechanisms by which Psd3 downregulation results in lower hepatic fat content, we examined expression levels of genes involved in triglyceride synthesis. Consistent with the in vitro experiments, $P s d 3$ downregulation in mice fed the NASH-inducing diet reduced the expression of genes involved in de novo lipogenesis (Fasn, Acc1. $S c d 1)$ (Fig. 7i-1). Psd3 downregulation also reduced hepatic expression levels of monocyte chemoattractant protein 1 (Ccl2), involved in monocyte infiltration and $\alpha$-smooth muscle actin (Acta2), a marker of myofibroblast formation (Fig. $7 \mathrm{~m}, \mathrm{n}$ ).
To confirm our in vivo data in another model, liver Psd3 levels were silenced using a GalNAc-conjugated Psd3 ASO in mice fed a choline-deficient high-fat diet (CD-HFD) for 12 weeks (Supplementary Figs. 8 and 9). As expected, the CD-HFD led to a reduction in body weight irrespective of the treatment. However, this reduction was recovered at the end of the treatment. Psd3 ASO treatment resulted in robust downregulation of liver Psd3 mRNA levels. Consistent with the diet-induced NASH model, Psd3 ASO treatment reduced the liver total amount of triglycerides and free cholesterol in the CD-HFD model (Supplementary Fig. 8). Moreover, Psd3 ASO treatment reduced the severity of liver steatosis (Supplementary Fig. 9). However, there were no differences observed in liver inflammation or CollA1 levels between the Psd3 and control ASO groups in this model (Supplementary Fig. 8).

\section{Discussion}

To date, single-nucleotide sequence variants in PNPLA3 (ref. ${ }^{14}$ ), TM6SF2 (ref. ${ }^{12}$ ), GCKR (ref. ${ }^{15}$ ), MBOAT7 (ref. ${ }^{13}$ ), HSD17B13 (ref. ${ }^{29}$ ), MARC1 (ref. ${ }^{30}$ ), APOE and GPAM ${ }^{31}$ have been shown to be associated with FLD. However, there is still a missing heritability for this disease ${ }^{16,17}$. Here, we describe a genetic locus, not previously linked to hepatic fat content, in PSD3 involved in the susceptibility to FLD in individuals at risk. Furthermore, downregulation of this gene resulted in protection against FLD in mice fed with a NASH-inducing diet, and in lower intracellular fat in human primary hepatocytes cultured in 2D and 3D.

Based on the fact that circulating triglycerides are a proxy for liver fat content $t^{19-23}$, we started by examining the association between common non-synonymous variants influencing circulating triglycerides ${ }^{18}$ and liver fat content in the DHS. Using this approach, we found three loci associated with liver fat content. Two of these were already well-known genetic determinants of FLD, 

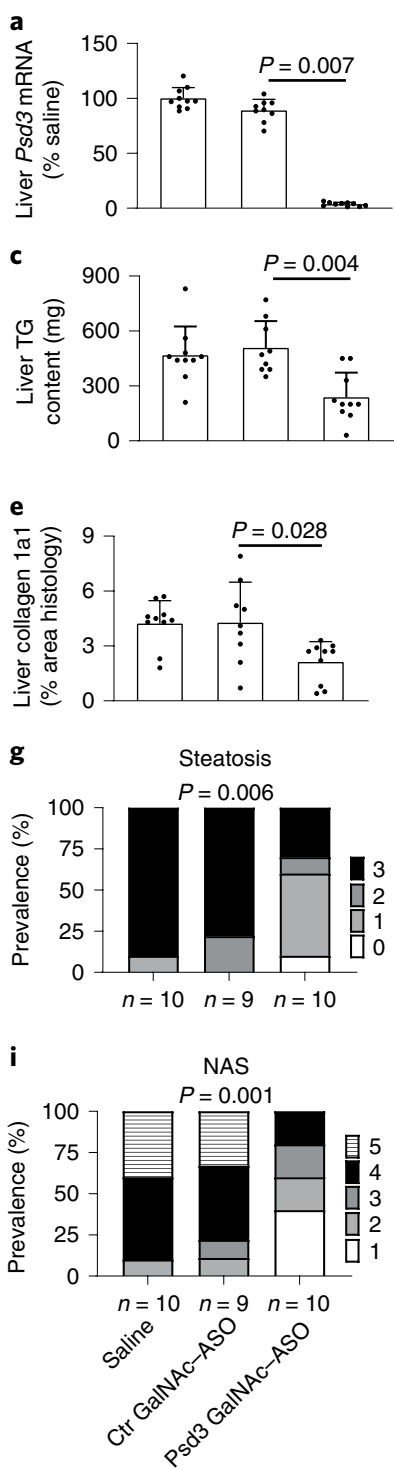

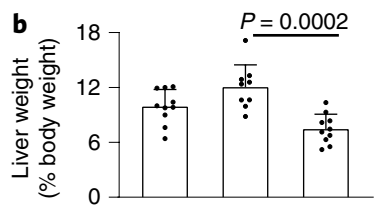

d

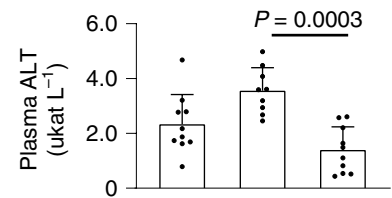

f

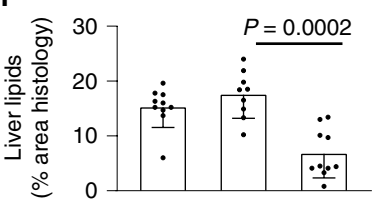

h

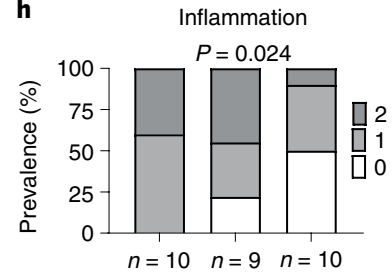

j

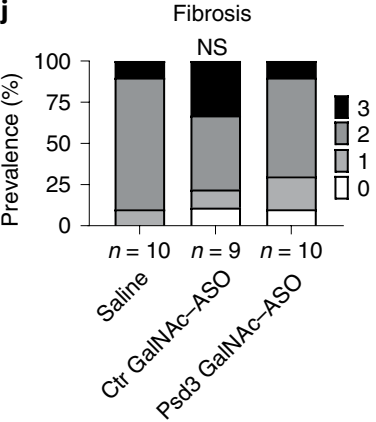

Fig. 6 | Liver Psd3 downregulation in mice fed a NASH-inducing diet reduced the severity of steatosis, inflammation and NAS, and liver collagen levels. C57BL/6 male mice were fed a NASH-inducing diet for a total of 50 weeks and during the last 16 weeks, groups of mice were dosed via once weekly subcutaneous injections with saline ( $n=10$ animals), control GalNAc-ASO ( $n=9$ animals, $5 \mathrm{mg}$ per kg body weight per week) or Psd3 GalNAc-ASO ( $n=10$ animals, $5 \mathrm{mg}$ per kg body weight per week). a-d, Psd3 GalNAc-ASO reduced liver Psd3 mRNA expression levels (a) and was associated with reduced liver weight (b), total liver triglyceride content (c) and plasma ALT levels (d). e,f, Psd3 GalNAc-ASO treatment reduced liver Col1a1 protein levels (e) and liver lipid droplet number (f). $\mathbf{g}-\mathbf{j}$, Psd3 GalNAc-ASO treatment reduced the severity of steatosis ( $(\mathbf{g})$, inflammation (h) and NAS (i), although there were no significant changes in liver fibrosis grade $(\mathbf{j})$. $\mathbf{a}-\mathbf{f}$, Data are presented as mean \pm s.d. Two-sided $P$ values were calculated by one-way ANOVA Kruskal-Wallis non-parametric test followed by Dunn's correction for multiple comparisons. Multiple comparisons were performed between the mean of each group and the mean of the control group (Ctr GalNAc-ASO). $\mathbf{g}-\mathbf{j}$, Data are presented as prevalence (\%) of the specified disease's degree. Colour shading from white to black indicates increased disease severity. Two-sided $P$ values were calculated by using ordinal regression analyses. NS, non-significant.

namely the TM6SF2 and GCKR, and the third was a dinucleotide polymorphism (rs71519934) in PSD3.

Next, we examined the association between the rs71519934 and liver disease in a cohort of 1,951 individuals at risk for liver disease with an available liver biopsy. In this cohort, we found that the rs71519934 186T allele was associated with a reduction in liver steatosis, inflammation, ballooning and fibrosis. Moreover, the rs71519934 was associated with lower circulating lipoprotein levels. To confirm our data, we examined the rs7003060 (in complete linkage disequilibrium $\left(D^{\prime}=1, r^{2}=1\right)$ with rs71519934) in European individuals $(n=10,970)$ from the UK Biobank with liver fat content measurement available, and found an association with reduced hepatic fat content in individuals with obesity. To further confirm our data, we examined the contribution of the rs71519934 to liver disease in a cohort of individuals with obesity at risk for liver disease from central Europe with liver biopsy available $(n=674)$. In this cohort we found that the rs71519934 was associated with protection against liver steatosis, inflammation and fibrosis. Meta-analyses of the two studies in which liver biopsy was available confirmed a robust effect of the genetic variant against liver steatosis, fibrosis and ballooning. A strength of the genetic study is the replication of the association in two independent cohorts with liver biopsy available; a limitation is that our validation and replication cohorts comprised only European adults and therefore further studies are warranted to 
a

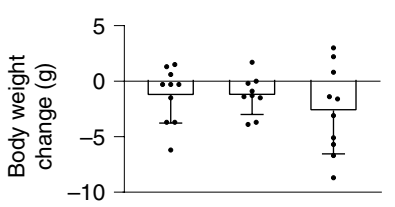

e

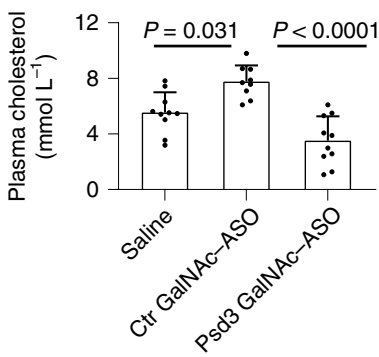

i

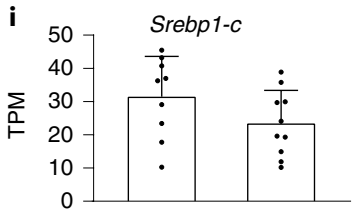

m

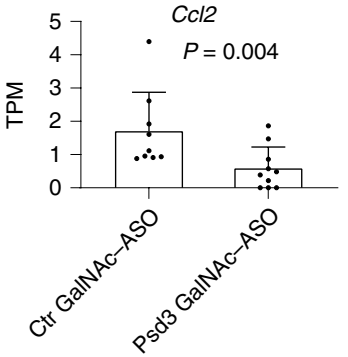

b

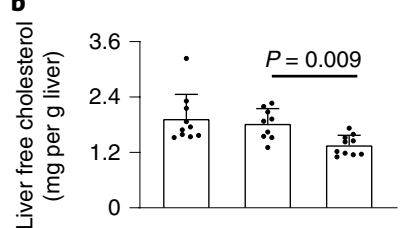

f

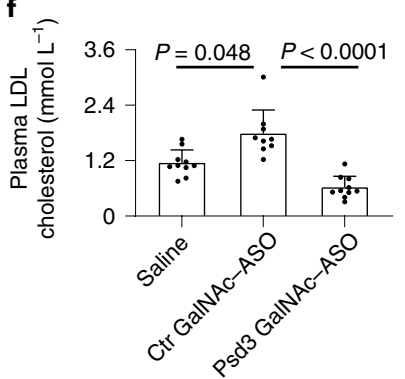

j

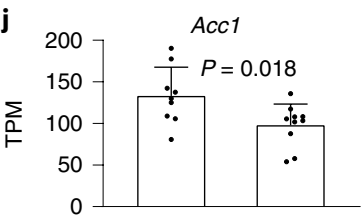

n

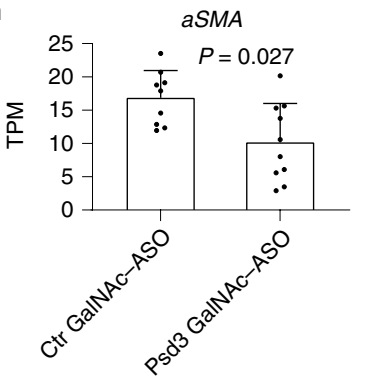

c

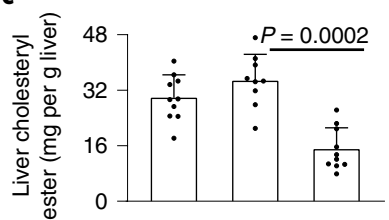

g

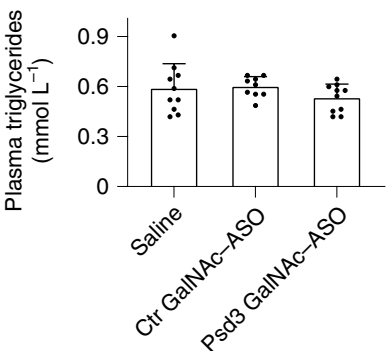

k

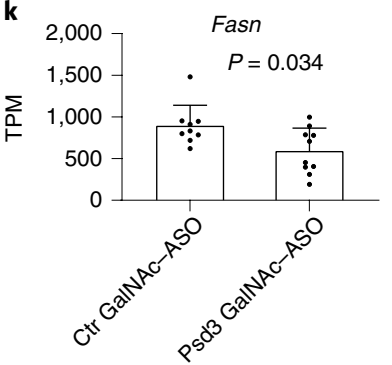

d

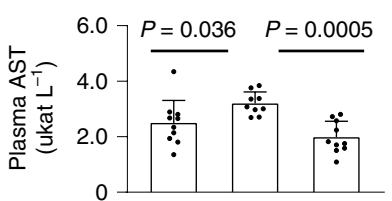

h

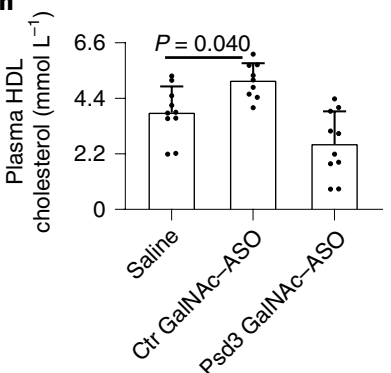

I

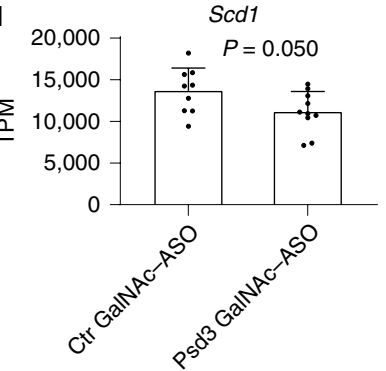

Fig. 7 | Liver Psd3 downregulation in mice fed a NASH-inducing diet reduced liver cholesterol and circulating LDL cholesterol levels and reduced expression of hepatic genes involved in de novo lipogenesis. $\mathrm{C} 57 \mathrm{BL} / 6$ male mice were fed a NASH-inducing diet for a total of 50 weeks and during the last 16 weeks, groups of mice were dosed via once weekly subcutaneous injections with saline ( $n=10$ animals), control GalNAc-ASO ( $n=9$ animals, 5 mg per $\mathrm{kg}$ body weight per week) or Psd3 GalNAc-ASO ( $n=10$ animals, $5 \mathrm{mg}$ per kg body weight per week). a-h, Psd3 GalNAc-ASO treatment did not affect body weight gain (a) but reduced liver cholesterol (b) and cholesteryl ester (c), plasma AST (d), total cholesterol (e) and LDL cholesterol levels (f). There were no effects on plasma triglyceride ( $\mathbf{g}$ ) or HDL cholesterol (h) levels. Hepatic mRNA was quantitated by digital gene expression profiling as described in the Methods and expressed as transcripts per million. i-n, Psd3 GalNAc-ASO treatment had no effect on hepatic Srebp1-c (i) but significantly reduced hepatic $\operatorname{Acc1}(\mathbf{j})$, Fasn (k), Scd1 (I), $\alpha$ Sma ( $\mathbf{m})$ and Ccl2 (n) mRNA expression levels. Data are presented as mean \pm s.d. a-h, Two-sided $P$ values were calculated by one-way ANOVA Kruskal-Wallis non-parametric tests followed by Dunn's multiple comparisons tests. Multiple comparisons were performed by comparing each group with the control group (Ctr GalNAc-ASO). i-n, Two-sided P values were calculated by Mann-Whitney non-parametric tests. TPM, transcripts per million.

test the contribution of this variant to liver disease in other ethnic groups and in children.

The index SNP (rs1495741) identified in the genome-wide association study on circulating triglycerides is an intergenic variant located between PSD3 and NAT2. We did not find any association of NAT2 missense gene variants with liver fat content. Moreover, there was no difference in the expression of NAT2 between individuals with or without FLD. These data suggest that PSD3 and not NAT2 is involved in FLD. Recently, the rs1495741 was associated at a genome-wide level with susceptibility to drug-induced liver injury ${ }^{32}$, reinforcing the concept of an involvement of this locus in liver disease.

PSD3, also known as EFA6D, EFA6R and HCA67, is a member of the EFA6 protein family. Knowledge about this protein is lim- ited, but proteins in this family have guanine nucleotide exchange activity for ARF6, a small guanosine triphosphatase that regulates endosomal trafficking and cytoskeleton remodelling ${ }^{33}$. PSD 3 was originally identified by gene expression screening on human hepatocellular carcinoma ${ }^{34}$. However, PSD3 has not previously been shown to be involved in hepatic lipid metabolism.

Next, we examined the PSD3 genotype effect on intracellular neutral lipid levels in human primary hepatocytes cultured in 2D. Consistent with the genetic association data, hepatocytes from a homozygous 186T donor had lower levels of intracellular lipids than homozygous 186L hepatocytes. PSD3 protein levels were elevated when increased amounts of OA were added to the cell cultures with either genotype. However, at any given OA concentration, hepatocytes homozygous for the $186 \mathrm{~T}$ allele had lower PSD3 protein levels 

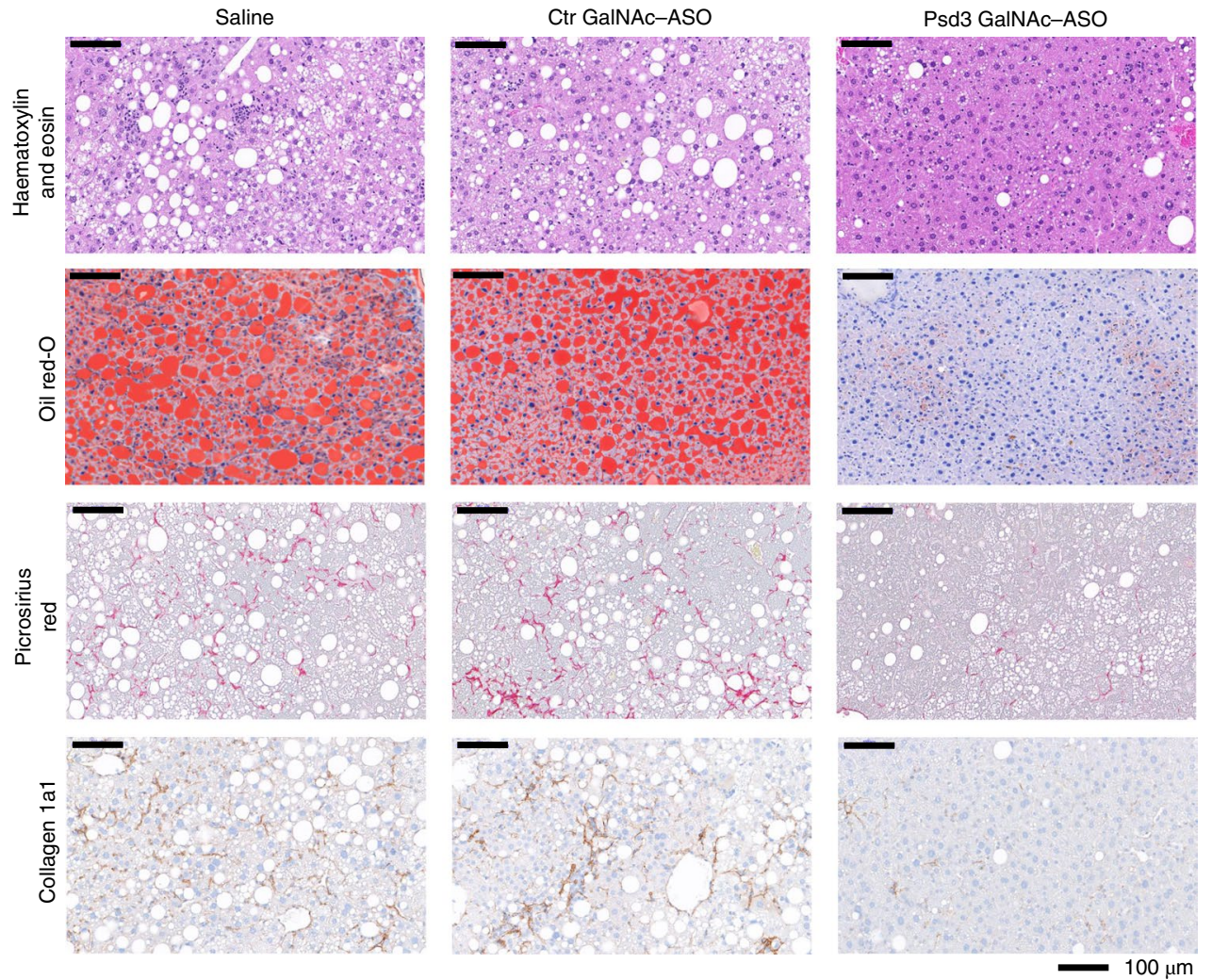

Fig. 8 | Liver Psd3 downregulation in mice fed a NASH-inducing diet improved liver histopathology. C57BL/6 male mice were fed a NASH-inducing diet for a total of 50 weeks and during the last 16 weeks, groups of mice were dosed via once weekly with subcutaneous injections of saline $(n=10$ animals), control GalNAc-ASO ( $n=9$ animals, $5 \mathrm{mg}$ per kg body weight per week) or Psd3 GalNAc-ASO ( $n=10$ animals, 5 mg per kg body weight per week). Liver sections were stained with haematoxylin and eosin, ORO or picrosirius red according to standard procedures. Consecutive sections were immunohistochemically stained for Col1a1. Representative pictures are presented. Ctr, control.

than $186 \mathrm{~L}$ hepatocytes. Transcriptomic analyses revealed that carriers of the 186T allele had lower expression of key genes involved in the de novo triglyceride and cholesterol biosynthesis.

To gain insight into the mechanisms underlying the genetic association, we examined the hepatic expression of PSD3 in individuals with or without FLD. Overall, PSD3 isoform-a was abundantly expressed in the liver. In addition, and consistent with primary human hepatocyte data, individuals with FLD had higher hepatic PSD3 mRNA expression levels compared with non-FLD controls. Based on this, we hypothesized that PSD3 downregulation would result in a reduction in intracellular liver fat content. Consistent with this hypothesis, downregulation of PSD3 resulted in a reduction in neutral lipids in primary human hepatocytes cultured in $2 \mathrm{D}$ and $3 \mathrm{D}$. The downregulation-induced reduction was present in both genotypes, except for $186 \mathrm{~T}$ in $3 \mathrm{D}$ in which only a trend was observed. Similar results were observed in human and rat hepatoma cells.

Radiolabelled tracer experiments in vitro showed that downregulation of $P s d 3$ resulted in a reduction in triglyceride synthesis with no differences in the utilization of fatty acids via beta oxidation. Consistent with the genetic associations with total and LDL cholesterol levels in humans, Apo B secretion was lower in these cells. This may be because of the primary reduction in lipid content due to the lower intracellular synthesis of triglycerides.

Moreover, in silico analyses of the L186T substitution (Supplementary Table 7) indicated that this amino acid change is tolerated. Taking all this together, we speculate that the L186T substitution in PSD3 induces partial loss of function of the protein, becoming apparent in individuals at risk for FLD.
To confirm our data in an in vivo model, we downregulated $P s d 3$ in mice fed a NASH-inducing diet. Consistent with the human genetic data, downregulation of $P s d 3$ resulted in protection against liver steatosis, inflammation and collagen deposition in these mice. We did not see any significant changes in liver fibrosis stage in these mice and this may be due to a lack of power to detect this association in the in vivo study. However, the amount of liver Collal visualized by histological staining was lower after downregulation of $P s d 3$. By using Mendelian randomization, we have shown that liver fat content per se is deleterious to the liver ${ }^{35}$ causing inflammation and fibrosis. The reduction in inflammation and collagen content observed in this study may be secondary to the reduction in liver fat content due to $P s d 3$ downregulation. To confirm our in vivo data, we silenced hepatic Psd3 in mice fed a CD-HFD. In these mice, $P s d 3$ downregulation resulted in a lower hepatic triglyceride content. However, no differences were observed in liver Collal deposits. This discrepancy could be because this experimental amino acid-deficiency model is very aggressive and develops liver fibrosis in a very short time frame. In addition, this model comes with a negative energy balance and weight loss rather than obesity and insulin resistance, thus the translational relevance of this model to a human NASH patient could be questioned.

To test whether de novo lipogenesis was perturbed in vivo, we examined expression data for the genes involved in triglyceride synthesis. Consistent with the in vitro data, downregulation of $P s d 3$ resulted in a reduction in key enzymes involved in triglyceride synthesis. What are the mechanisms linking PSD3 to triglyceride synthesis? Recently, we showed that hepatic Mboat7/LYPAAT1 inactivation results in an increase in hepatic triglyceride synthesis ${ }^{36}$. 
This increase was due to an excess of triglyceride precursors deriving from a non-canonical pathway related to a high phosphatidylinositol turnover. PSD3 is an activator of ARF6, which in turn, is one of the activators of phospholipase $\mathrm{D}$. This lipase hydrolyses phosphatidylcholine to phosphatidic acid and choline. Phosphatidic acid is a main precursor for triglycerides synthesis in the liver. PSD3 downregulation resulted in a reduction in ARF6 activation. Taking all this together, we speculate that lower ARF6 activation due to PSD3 downregulation results in less substrate being available for triglyceride synthesis in the liver.

In summary, PSD3 rs71519934 was associated with protection against FLD in individuals at risk and $P S D 3 / P s d 3$ downregulation led to a reduction in hepatocellular fat content in human primary hepatocytes and in human and rodent hepatoma cells. Furthermore, liver $P s d 3$ silencing resulted in protection against high liver fat, inflammation and collagen content in vivo in mice. Further studies are warranted for further mechanistic understanding and to evaluate the potential of this target to treat FLD.

\section{Methods}

Selection of gene loci. The study design is shown in Supplementary Fig. 1. In the discovery phase, 32 genetic tag SNPs previously associated with triglycerides as the main $(n=24)$ or secondary trait $(n=8)$ at a genome-wide significance level were selected ${ }^{18}$ (Supplementary Table 1). All missense and nonsense variants within $50 \mathrm{~kb}$ of the selected loci that were on the Illumina HumanExome BeadChip (including the tag SNPs when available) were then tested for their association with liver fat content in 2,736 participants in the DHS. Liver fat content was measured using proton magnetic resonance spectroscopy. When the tag SNP was intergenic, variants in both genes adjacent to the tag SNP were examined.

Identification of the PSD3 genetic variant. Among the genetic variants tested, three had a MAF $>5 \%$ in Europeans from the 1000 Genome Project (dbSNP, Current Build 154, released 21 April 2020) and a nominally significant association with liver fat content in the DHS. Among these variants, TM6SF2 rs58542926 and GCKR rs1260326, two well-known genetic variants associated with the entire spectrum of FLD as well as with circulating triglycerides ${ }^{15,24}$, were associated with increased liver fat content. The other variant, namely the rs71519934 (found as rs7003060 that is in complete linkage disequilibrium $\left(D^{\prime}=1, r^{2}=1\right)$ in the PSD3 gene, was associated with lower liver fat content.

In the LBC, the rs71519934 had an overall MAF of 33\%. When we stratified the cohort based on the centre of recruitment, we found that the rs71519934 had a higher frequency in individuals from Finland (MAF $48 \%$ and $42 \%$ in the two subgroups from Finland) than in participants from Italy (MAF 26\% and 28\% in the two subgroups from Italy; Supplementary Table 8) reflecting reference data (Exome Aggregation Consortium database). The genotype frequencies were in HWE in three-quarters of subcohorts (Supplementary Table 8). The deviation in the Milan subcohort may be because this subcohort is a selected population of individuals at risk for liver disease.

Owing to these differences in the MAF, we included the centre of recruitment as a covariate in subsequent multivariate analyses. To understand the effect of the genetic variant, we examined the histological degree of the different components of liver disease, namely, steatosis (the histological measurement of liver fat content), inflammation, ballooning (an index of cell damage) and fibrosis (the main predictor of mortality related to liver disease).

Study cohorts. Dallas Heart Study. The DHS is a multi-ethnic population-based sample of Dallas County residents in which hepatic triglyceride content was measured with proton magnetic resonance spectroscopy (supplementary material) in a subset of 2,736 individuals $s^{37,38}$. For the current study, only individuals with a measurement of hepatic triglyceride content have been included. All analyses were based on cross-sectional data. The study was approved by the institutional review board of University of Texas Southwestern Medical Centre, and all individuals provided written informed consent.

Liver Biopsy Cohort. The LBC is a cross-sectional study of individuals of European descent who underwent liver biopsy for suspected NASH or severe obesity, consecutively enrolled in three European centres ${ }^{13,24}$. In addition, a total of 323 individuals from a fourth and independent Finnish centre $\mathrm{s}^{39}$ have been included (supplementary material) ${ }^{40-42}$. In the current study, only individuals with complete data available for both PSD3 genotype and liver histology were included $(n=1,951)$. The diagnosis of NASH was based on the presence of steatosis with lobular necro-inflammation and ballooning or fibrosis. Disease activity was assessed according to the NAS; fibrosis was staged according to the recommendations of the FLD clinical research network ${ }^{28}$. Scoring of liver biopsies was performed blind by pathologists unaware of the patient's genotype and status. The study was approved by the ethics committees of the Fondazione IRCCS Ca' Granda (Milan), Palermo University Hospital (Palermo), Northern Savo Hospital District in Kuopio (Finland), and the ethics committee of the Hospital District of Helsinki and Uusimaa (Finland).

UK Biobank. The UK Biobank is a large cohort study comprising more than 500,000 adults (aged between 40 and 69 years at recruitment) who visited 22 recruitment centres throughout the UK between 2006 and 2010. Both the phenotypic and genotypic data used in the current study were obtained from the UK Biobank under Application Number 37142.

For the current study, we restricted our analysis to a subset of unrelated white British participants from the UK Biobank, after further removal of individuals with more than ten putative third-degree relatives, a mismatch between self-reported and genetically inferred gender, putative sex chromosome aneuploidy, withdrawn consent and those who were identified by the UK Biobank as outliers based on heterozygosity and missingness ${ }^{43,44}$.

We used liver MRI-derived PDFF data (data field 22,436). Briefly, participants were scanned with a Siemens MAGNETOM Aera 1.5-T MRI scanner using a 6minute dual-echo Dixon Vibe protocol, and a single multi-echo slice was further acquired to analyse the liver $\mathrm{PDFF}^{45,46}$.

The UK Biobank study received ethical approval from the National Research Ethics Service Committee North West Multi-Centre Haydock (reference 16/ $\mathrm{NW} / 0274)^{47,48}$

The central European independent replication cohort. In total, 674 obese adult Europeans at risk for FLD who underwent percutaneous or surgical liver biopsy were included (supplementary material). NASH was defined by the NAS. The presence of fibrosis was assessed histologically according to the Kleiner classification ${ }^{28}$. Liver biopsies were read by two experienced histopathologists in a blinded fashion. All patients gave their written informed consent for liver biopsy and genetic testing.

Genotyping. All participants from the DHS were previously genotyped for the variants using an Illumina Infinium HumanExome BeadChip ${ }^{12,14}$.

Participants from the LBC and from the central European independent replication cohort were genotyped by TaqMan $5^{\prime}$ nuclease assays (Life Technologies). The allelic discrimination probe for PSD3 rs71519934 was not commercially available. A custom assay for this variant has been designed (supplementary material).

Nine individuals denoted as AC homozygotes $(n=3)$, CT homozygotes $(n=3)$ and AC/CT heterozygous $(n=3)$ by the TaqMan assay were Sanger sequenced with consistent results (Supplementary Fig. 2)

UK Biobank participants were genotyped using two highly similar UK BiLEVE or UK Biobank Axiom arrays (>95\% overlap). Genotyped data were then imputed based on the 1000 Genomes Phase 3, UK10K haplotype and Haplotype Reference Consortium reference panels ${ }^{47}$. Genotype data for the rs71519934 dinucleotide change were not available in the UK Biobank; the rs 7003060 (identifying the first nucleotide change of the rs71519934) was among directly genotyped variants and has been used instead.

Gene expression analysis. Gene expression in human liver biopsies. For human liver biopsies, mRNA expression of the different PSD3 isoforms, and of PSD3 and NAT2 in FLD versus non-FLD was measured in 77 participants from the Milan subset of the LBC (supplementary material) ${ }^{35,49}$. Informed consent was obtained from each participant. The study protocol was approved by the ethics committee of the Fondazione IRCCS Ca' Granda, Milan, and conformed to the ethical guidelines of the 1975 Declaration of Helsinki.

Gene expression in primary and immortalized cells. For primary and immortalized cells, gene expression was assessed by real-time quantitative polymerase chain reaction (quantitative retro transcription PCR) and analysed using the $2^{-\Delta \Delta C t}$ method (supplementary material).

Gene expression in mice. Liver RNA was purified and subjected to quantitative PCR analysis (supplementary material) ${ }^{50}$

Psd3 mRNA was quantitated using the primer probe set Mm01351099_m1 (ThermoFisher Scientific). RNA transcript levels were normalized to total RNA levels using Quant-iT RiboGreen RNA reagent (ThermoFisher Scientific) ${ }^{51}$.

For lipogenic and inflammatory gene expression, total RNA was sequenced on a NextSeq500 sequencing instrument (Illumina) (supplementary material).

In vitro 2D cell culture and quantification of intracellular fat. Cryopreserved primary human hepatocytes were purchased from BioIVT and LONZA. After genotyping, cells were identified as homozygous for 186L (BioIVT lot number: BGW-M00995 (male, aged 50 years, BMI 20.4)) and homozygous for 186T (LONZA lot number: HUM183001 (female, aged 20 years, BMI 28.9)). Rat hepatoma McArdle (McA-RH7777 cells, homozygotes for $180 \mathrm{~T}$ that corresponds to 186T in human PSD3 according to the alignment of human NP_056125.3 and rat 
XP_017455908.1) were from ATCC and human hepatocytes Huh7 (PSD3 L186L) were from the JCRB cell bank.

In basal conditions, primary cells were cultured with media provided by the respective companies, McA-RH7777 cells were cultured in DMEM containing $10 \%$ foetal bovine serum (FBS), and Huh7 were cultured in DMEM (low glucose) containing $10 \%$ FBS

In experimental conditions, $24 \mathrm{~h}$ after seeding, cells were transiently transfected with $30 \mathrm{nM}$ SCR siRNA (AM4611; ThermoFisher Scientific), human PSD3 siRNA (mix of s23653, s23654 and s23655 for primary and Huh7 cells; ThermoFisher Scientific), rat Psd3 siRNA (mix of s157480, s157481 and s157482 for McA-RH7777 cells; ThermoFisher Scientific) or human ARF6 siRNA (mix of s1565, s1566 and s1567 for Huh7 cells; ThermoFisher Scientific) by Lipofectamine 3000 transfection reagent (L3000-075; ThermoFisher Scientific) according to the manufacturer's instructions, and grown in serum-free regular medium supplemented with $10 \mu \mathrm{M}$ (primary cells), $50 \mu \mathrm{M}$ (McA-RH7777) or $25 \mu \mathrm{M}$ (Huh7) OA for $48 \mathrm{~h}$.

Intracellular neutral fat content was visualized by ORO staining and images were acquired using an Axio KS 400 Imaging System and AxioVision v.4.8 software (Zeiss) at $\times 100$ magnification. The ORO-stained area was quantified using Biopix iQ software v.2.3.1 (refs. ${ }^{52,53}$ ).

RNA-seq and differentially expressed genes comparison. Human primary hepatocytes from donors homozygous for the $186 \mathrm{~T}$ or $186 \mathrm{~L}$ allele were cultured in $2 \mathrm{D}$ in their respective media. Total RNA was extracted and its purity and integrity number were evaluated (supplementary material). Illumina sequencing and subsequent analysis was carried out by Novogene (UK) (supplementary material). Differential expression analysis between two conditions/groups (two biological replicates per condition) was performed using the DESeq2 $\mathrm{R}$ package (v.2_1.6.3). The resulting two-sided $P$ values were adjusted using the Benjamini and Hochberg's approach for controlling the false discovery rate (FDR). Genes with an adjusted $P$ value $<0.05$ were assigned as differentially expressed.

\section{D liver spheroid formation and quantification of intracellular fat.}

Cryopreserved primary human hepatocytes from two different donors (as described above) were used to generate spheroids. Cells were seeded into ultralow attachment 96-well plates (Corning) (supplementary material) ${ }^{54}$ and transfected with negative control SCR siRNA or PSD3 siRNA as described above. The plates were then centrifuged at $100 \mathrm{~g}$ for $5 \mathrm{~min}$. Once the cells have collected at the bottom, self-aggregation leads to the formation of spheroids. Spheroids were cultured for 7 days (supplementary material) and their ATP-based viability was determined using a CellTiter-Glo Luminescent Cell Viability Assay kit (Promega). Cellular ATP was normalized to spheroid volume. Images of the spheroids were taken using an Axio Vert.Al inverted microscope (Carl Zeiss $A G)$. For quantification of neutral fat, spheroids were fixed and stained as described previously ${ }^{55}$. The ORO-stained area was normalized to the number of 4,6-diamidino-2-phenylindole-stained nuclei and quantified using Image J (v.1.52h, NIH)

PSD3 polyclonal antibody generation. Human PSD3 (Uniprot Q9NYI01) amino acids D527-T1048 were expressed in Sf21 cells in fusion with an $\mathrm{N}$-terminal $6 \times$ His tag (separated by a flexible linker) from the pFastBacl vector (ThermoFisher Scientific). His-PSD3 was purified under endotoxin-free conditions by immobilized metal affinity chromatography on a nickel column (GE Healthcare) and size-exclusion chromatography on a Superdex 200 column (GE Healthcare) in $2 \times$ PBS buffer $\mathrm{pH}$ 7.4. Antibody generation was carried out by Antibody Applications. Purified His-PSD3 (D527-T1048) was used to immunize three male 22-week-old New Zealand white rabbits, using a standard 90-day protocol. Anti-PSD3 IgG from each animal was affinity purified from final bleed rabbit serum on an NHS-activated Sepharose 4 fast flow agarose column (Cytiva) coupled to His-PSD3. A lead antibody was validated via silencing of endogenously expressed PSD3 in human hepatoma HepaRG cells (Supplementary Fig. 10).

Immunoblotting. Proteins were separated by SDS-PAGE and transferred to nitrocellulose membranes in accordance with standard procedures. Protein lysates were prepared from cell extracts using M-PER protein extraction reagent (ThermoFisher Scientific) supplemented with $10 \%$ protease inhibitor (SIGMAFAST; Sigma-Aldrich). Primary antibodies used were: anti-PSD3 custom antibody (dilution 1:1,000, as described above), rabbit anti-calnexin (dilution 1:1,000; Sigma-Aldrich) and mouse monoclonal anti-Arf6 (dilution 1:1,000, provided with the Arf6 activation kit, Cell Biolabs Inc.). Blots were probed with primary antibodies followed by the appropriate horseradish peroxidase-conjugated secondary antibody (dilution 1:2,000) and developed using ECL substrate (Immobilon Western Chemiluminescent HRP Substrate; Merck Millipore).

ARF6 activation assay. A gamma adaptin ear-containing ARF binding 3 family protein-binding domain (GGA3 PBD) pull-down assay was performed using an ARF6 activation assay kit (STA-407-6, Cell Biolabs) according to the manufacturer's protocol. Briefly, Huh7 cells were transfected with either SCR siRNA, PSD3 siRNA as described above, or with ARF6 siRNA (mix of s1565, s1566 and s1567, ThermoFisher Scientific) for $48 \mathrm{~h}$ (supplementary material). Activated ARF6 was then pulled down using GGA3 PBD agarose beads (supplementary material) and along with the corresponding total cell fraction proteins, was analysed by immunoblotting for ARF6 and calnexin.

PSD3 and active ARF6 immunohistochemistry. Immunostaining for PSD3 and active ARF6 was performed on formalin-fixed and paraffin-embedded liver specimens from eight patients with NAFLD from the LBC $(n=4$ heterozygotes (L186T) and $n=4$ homozygotes for the 186L allele of the PSD3 gene). The automated Ventana BenchMark ULTRA IHC/ISH Staining Module (Ventana) was used together with the Ultraview 3,3'-diaminobenzidine v.1 detection system (Ventana). Tissue sections $(4 \mu \mathrm{m})$ were deparaffinized $\left(\right.$ at $\left.72^{\circ} \mathrm{C}\right)$ and incubated in EZPrep Volume Adjust (Ventana). At intervals, steps slides were washed with a cell conditioning solution, Cell Conditioner 1, Tris-EDTA-based buffer, pH 7.8. For analysis of PSD3, sections were first incubated with ultraviolet inhibitor blocking solution for $4 \mathrm{~min}$ at $36^{\circ} \mathrm{C}$, then with the protease 1 for $4 \mathrm{~min}$ at $36^{\circ} \mathrm{C}$, and finally with anti-PSD3 primary antibody (29-749, dilution 1:100; ProSci) for $1 \mathrm{~h}$ at room temperature. For analysis of ARF6, a heat-induced antigen retrieval protocol set for $56 \mathrm{~min}$ at $91^{\circ} \mathrm{C}$ was carried out using a Tris-EDTA-boric acid, $\mathrm{pH}$ 8.4, buffer (Cell Conditioner 1). Sections were incubated with ultraviolet inhibitor blocking solution for $4 \mathrm{~min}$ and then with anti-activated ARF6 primary antibody (26918, dilution 1:200; NewEast Biosciences) for $1 \mathrm{~h}$ and $4 \mathrm{~min}$ at room temperature. For both analyses, incubation with horseradish peroxidase-linked secondary antibody (8 min) (1706515 for PSD3, dilution 1:100, and 170-6516 for active ARF6, dilution 1:150; both Bio-Rad) was followed by incubation with $3,3^{\prime}$-diaminobenzidine chromogen and substrate $(8 \mathrm{~min})$ and copper enhancer $(4 \mathrm{~min})$. Counterstain (haematoxylin) was applied for $12 \mathrm{~min}$ before 4 -min incubation with bluing reagent.

Stained tissue sections were analysed under an optical microscope by a pathologist who was blinded to the PSD3 genotype.

De novo triglyceride synthesis. De novo triglyceride synthesis was analysed using ${ }^{3} \mathrm{H}$-glycerol ${ }^{56}$. Briefly, McA-RH7777 and Huh7 cells were seeded in triplicate in six-well plates. Twenty-four hours after seeding, cells were transfected with $P s d 3$ siRNA or SCR siRNA for $48 \mathrm{~h}$ in regular medium (supplementary material). Forty-eight hours after transfection, cells were incubated with ${ }^{3} \mathrm{H}$-glycerol (PerkinElmer) plus OA for 15, 30 or $60 \mathrm{~min}$. Cell lysates were collected and lipids were extracted (supplementary material) ${ }^{57}$, separated and quantified by thin-layer chromatography. Spots corresponding to triglycerides were visualized with iodine vapour and added to vials with scintillation fluid. Radioactivity was measured using a scintillation counter as disintegrations per min.

Apolipoprotein B secretion. Apo B secretion in vitro was measured by adapting the previously described protocol ${ }^{53}$. Briefly, McA-RH7777 cells were transfected with $P s d 3$ siRNA or SCR siRNA. Forty-eight hours after transfection, cells were labelled with ${ }^{35} \mathrm{~S}$ Met/Cys (PerkinElmer) and chased for 5, 15, 30 or $60 \mathrm{~min}$ (supplementary material). Apo B was then immunoprecipitated (supplementary material) and subsequently separated on 3-8\% gradient SDS-PAGE. The gel was then dried, exposed overnight on BAS-MS film and visualized using Phosphoimager (Fujifilm FLA-3000).

Beta oxidation. Beta oxidation was analysed using ${ }^{3} \mathrm{H}$-palmitate ${ }^{58}$. Briefly, McA-RH7777 cells were transfected with Psd3 siRNA or SCR siRNA as described above. Forty-eight hours after transfection, cells were labelled with ${ }^{3} \mathrm{H}$-palmitate (PerkinElmer). Labelled palmitate from the cell media was then precipitated (supplementary material). The supernatant was collected and radioactivity was measured using a scintillation counter as disintegrations per min.

Liver Psd3 silencing in mice. ASO synthesis. Chimeric 16-mer phosphorothioate ASOs containing $2^{\prime}, 4^{\prime}$-constrained $2^{\prime}$-O-ethyl at positions 1-3 and 14-16, and a triantennary galactosamine (GalNAc) attached to the $5^{\prime}$-end of the ASOs were synthesized at Ionis Pharmaceuticals, as described previously ${ }^{59}$. Two different Psd 3 ASOs were used in the two studies; Psd3 ASO (5'-GTATTAATACTCTCTC-3') for the diet-induced NASH study, and Psd3 ASO (5'-CTTGATCGAGAATCCT-3') for the CD-HFD study. The effects were compared with mice dosed with a control ASO targeting no known murine gene (5'-GGCCAATACGCCGTCA-3'). The in vivo studies in mice complied with ethical regulations and were approved by an institutional animal care and use committee, and the Ionis local ethics committee (AAALAC Accreditation \#P-0305). All mice were obtained from the Jackson Laboratory and were housed in cages on a $12: 12 \mathrm{~h}$ light/dark cycle at $22.5 \pm 2.5^{\circ} \mathrm{C}$ and $50 \pm 20 \%$ humidity, fed ad libitum and had free access to drinking water for the duration of the studies.

Diet-induced NASH and ASO treatment. Six-week-old male C57BL/6 mice (homozygotes for 147T which corresponds to 186T in human PSD3 according to the alignment of human NP_056125.3 and mouse XP_017168192.1) were fed a NASH-inducing diet (D16010101, Research Diets) for 34 weeks. This NASH diet contains $40 \%$ of the kilocalories as fat (corn oil), $20 \%$ of the kilocalories as fructose and $2 \%$ cholesterol, and has previously been shown to accelerate NASH 
and liver fibrosis progression in mice ${ }^{60}$. Mice were bled and randomized into study groups based on body weight and plasma ALT levels ( $n=9-10$ per group). Mice were maintained on the NASH diet and treated with either saline $(n=10$ animals), control GalNAc-ASO ( $n=9$ animals, $5 \mathrm{mg}$ per kg body weight per week) or Psd3 GalNAc-ASO ( $n=10$ animals, $5 \mathrm{mg}$ per kg body weight per week) for 16 weeks via weekly subcutaneous injection. During the ASO treatment, body weight was monitored weekly. Seventy-two hours after the final ASO dose, mice were anaesthetized, blood was collected via cardiac puncture, and tissues were collected and either snap frozen in liquid nitrogen or fixed in formalin for histological analyses. Blood was centrifuged at $3,000 \mathrm{~g}$, and plasma was collected. The plasma and snap-frozen tissues were stored at $-80^{\circ} \mathrm{C}$.

CD-HFD and ASO treatment. Six-week-old male C57BL/6 mice were administered a weekly subcutaneous injection of either saline, control GalNAc-ASO or a Psd3 GalNAc-ASO at a dose of $5 \mathrm{mg}$ per kg body weight per week for 14 weeks $(n=12$ per group, 15 injections). For the first two weeks of treatment (three injections), all mice were fed a chow diet (5V12; LabDiet), and thereafter the mice were switched to a choline-deficient L-amino acid defined high-fat diet (A06071302; Research Diets) for the remaining 12 weeks of ASO treatment (12 injections). A smaller group of mice $(n=4)$ were kept on chow diet and dosed with saline for the entire study. During the ASO treatment period, body weight was monitored weekly. Seventy-two hours after the last ASO dose, mice were anesthetized, blood was collected via cardiac puncture and tissues were collected and either snap frozen in liquid nitrogen or fixed in formalin for histology analyses. Blood was spun at $3,000 \mathrm{~g}$ and plasma was collected. Plasma and snap-frozen tissues were stored at $-80^{\circ} \mathrm{C}$.

Plasma and liver biochemistry. Plasma transaminases (AST, ALT), total plasma cholesterol, plasma triglycerides, LDL and HDL cholesterol were quantitated using an Olympus clinical analyser (Beckman Coulter). Liver triglycerides, free cholesterol and cholesteryl ester were quantitated as described previously ${ }^{61}$.

Histopathology and image analysis. Snap-frozen liver samples were cut $(6 \mu \mathrm{m}$ thick) and stained with ORO according to standard procedures. After formalin fixation, dehydration and paraffin embedding, $4-\mu \mathrm{m}$ sections were stained with haematoxylin and eosin and picrosirius red according to standard procedures. Consecutive sections were immunohistochemically stained for Mac2 (CL8942AP; Cedarlaine) and Collal (LS-C343921; BioSite) in an automated Ventana Ultra system (Ventana Medical Systems). Image analysis was performed on digital images using Visiopharm Integrator System software (v.2018.09). Lipid-filled vacuoles, liver steatosis, inflammation, the NAS and the fibrosis stage were evaluated in the haematoxylin and eosin- and picrosirius red-stained liver sections according to the methods reported by Kleiner et $\mathrm{a}^{28}$. All histological assessments were performed blind by a board-certified veterinary pathologist.

Statistical analysis. For the DHS, the $P$ values for associations between liver fat content and the target variants were calculated using linear regression analysis adjusted for age, gender and the four leading principal components of ancestry. An additive genetic model was used in all analyses.

For the LBC and for the central European independent replication cohort, the association between the PSD3 rs71519934 variant and liver disease was evaluated under an additive genetic model by binary logistic (prevalence of liver disease) or ordinal regression (liver histological features) analysis adjusted for age, gender, BMI, centre of recruitment and number of PNPLA3 I148M mutant alleles. All analyses were performed using IBM SPSS statistics v.27.

For the UK Biobank, liver PDFF was first rank-based inverse normal transformed, and then the association with PSD3 rs7003060 was examined using linear regression adjusted for age, gender, BMI, the first ten genomic principal components and array type under an additive genetic model using R v.3.6.1, MATLAB R2020b (academic license).

For descriptive statistics, data are shown as the mean and s.d. or the median and interquartile range (i.q.r.) as appropriate. Categorical traits are shown as numbers and proportions. For continuous traits, $P$ values were calculated by linear regression under an additive genetic model unadjusted or adjusted for age, gender and BMI. Non-normally distributed traits were log-transformed before being entered into the model. For categorical traits, $P$ values were calculated by the chi-squared test or by binary logistic regression adjusted for age, gender and BMI using IBM SPSS statistics v.27.

Differences in PSD 3 and NAT2 expression levels in human tissues were evaluated by the Mann-Whitney non-parametric test (for comparisons between healthy individuals and those with FLD) or by linear regression analysis (for comparisons among genotypes).

Differential gene expression analysis in primary cells between two conditions/ groups (two biological replicates per condition) was performed using the DESeq2 R package (v.2_1.6.3) as described above ('RNA-seq and differentially expressed gene comparison'). The resulting two-sided $P$ values were adjusted using the Benjamini and Hochberg's approach for controlling the FDR.

For meta-analyses of the histological cohorts, an inverse variance meta-analysis of the two studies (LBC and central European replication cohort) was performed using package 'meta' with fixed and random effect models in R v.3.6.1 (ref. ${ }^{62}$ ).
For in vivo and in vitro studies, data are shown as the mean and s.d. $P$ values were calculated by the Mann-Whitney non-parametric test (in vitro) or one-way analysis of variance (ANOVA) Kruskal-Wallis non-parametric test with Dunn's correction for multiple comparisons (in vivo) using GraphPad Prism v.9. The severity scores of liver disease in the in vivo mouse studies were analysed by using ordinal regression analyses. All the reported $P$ values are two-sided.

Reagent or resource. The catalogue numbers and providers of reagents and resources are detailed in supplementary information.

Ethical approval. Our research complies with the principles outlined in the Declaration of Helsinki. The specific board/committee and institution that approved each protocol are listed in the relevant section. Each subject provided written informed consent.

Reporting Summary. Further information on research design is available in the Nature Research Reporting Summary linked to this article.

\section{Data availability statement}

All data associated with this study are present in the paper or the Supplementary Information. For UK Biobank, all individual-level phenotype/genotype data are accessible via a formal application to the UK Biobank http://www.ukbiobank.ac.uk. Antisense oligonucleotides associated with this study can be made available, on reasonable request, to academic researchers under a material transfer agreement with AstraZeneca and Ionis Pharmaceuticals. Owing to study participants' privacy data protection, the RNA-seq data of the liver biopsies of the LBC can only be made available on request to the corresponding authors for collaborative projects. Bulk RNA-seq data of the primary human hepatocytes are deposited in the NCBI SRA under the BioProject identifier PRJNA778044. All other data are available from the authors on reasonable request. The following online databases have been used: Database of Single Nucleotide Polymorphisms (dbSNP) (https://www. ncbi.nlm.nih.gov/snp/); Ensembl (https://www.ensembl.org/index.html); Exome Aggregation Consortium [ExAC] database (http://exac.broadinstitute.org). Source data are provided with this paper.

\section{Code availability statement}

Source data are provided with this paper. All codes and scripts used for association studies are available on request. Codes used for image analysis available on request.

Received: 23 August 2021; Accepted: 8 December 2021; Published online: 31 January 2022

\section{References}

1. Younossi, Z. \& Henry, L. Contribution of alcoholic and nonalcoholic fatty liver disease to the burden of liver-related morbidity and mortality. Gastroenterology 150, 1778-1785 (2016).

2. Eslam, M. et al. A new definition for metabolic dysfunction-associated fatty liver disease: An international expert consensus statement. J. Hepatol. 73, 202-209 (2020).

3. Estes, C. et al. Modeling NAFLD disease burden in China, France, Germany, Italy, Japan, Spain, United Kingdom, and United States for the period 2016-2030. J. Hepatol. 69, 896-904 (2018).

4. Armstrong, M. J. et al. Liraglutide safety and efficacy in patients with non-alcoholic steatohepatitis (LEAN): a multicentre, double-blind, randomised, placebo-controlled phase 2 study. Lancet 387, 679-690 (2016).

5. Neuschwander-Tetri, B. A. et al. Farnesoid X nuclear receptor ligand obeticholic acid for non-cirrhotic, non-alcoholic steatohepatitis (FLINT): a multicentre, randomised, placebo-controlled trial. Lancet 385, 956-965 (2015).

6. Pelusi, S. \& Valenti, L. Hepatic fat as clinical outcome and therapeutic target for nonalcoholic fatty liver disease. Liver Int 39, 250-256 (2019).

7. Eslam, M., Valenti, L. \& Romeo, S. Genetics and epigenetics of NAFLD and NASH: clinical impact. J. Hepatol. 68, 268-279 (2018).

8. Loomba, R. et al. Heritability of hepatic fibrosis and steatosis based on a prospective twin study. Gastroenterology 149, 1784-1793 (2015).

9. Makkonen, J., Pietilainen, K. H., Rissanen, A., Kaprio, J. \& Yki-Jarvinen, H. Genetic factors contribute to variation in serum alanine aminotransferase activity independent of obesity and alcohol: a study in monozygotic and dizygotic twins. J. Hepatol. 50, 1035-1042 (2009).

10. van Beek, J. H. et al. Heritability of liver enzyme levels estimated from genome-wide SNP data. Eur. J. Hum. Genet. 23, 1223-1228 (2015).

11. Vinkhuyzen, A. A., Wray, N. R., Yang, J., Goddard, M. E. \& Visscher, P. M. Estimation and partition of heritability in human populations using whole-genome analysis methods. Annu. Rev. Genet. 47, 75-95 (2013).

12. Kozlitina, J. et al. Exome-wide association study identifies a TM6SF2 variant that confers susceptibility to nonalcoholic fatty liver disease. Nat. Genet. 46 , 352-356 (2014) 
13. Mancina, R. M. et al. The MBOAT7-TMC4 variant rs641738 increases risk of nonalcoholic fatty liver disease in individuals of European descent. Gastroenterology 150, 1219-1230 (2016).

14. Romeo, S. et al. Genetic variation in PNPLA3 confers susceptibility to nonalcoholic fatty liver disease. Nat. Genet. 40, 1461-1465 (2008)

15. Speliotes, E. K. et al. Genome-wide association analysis identifies variants associated with nonalcoholic fatty liver disease that have distinct effects on metabolic traits. PLoS Genet. 7, e1001324 (2011).

16. Carlsson, B. et al. Review article: the emerging role of genetics in precision medicine for patients with non-alcoholic steatohepatitis. Aliment. Pharmcol. Ther. 51, 1305-1320 (2020).

17. Romeo, S., Sanyal, A. \& Valenti, L. Leveraging human genetics to identify potential new treatments for fatty liver disease. Cell Metab. 31, 35-45 (2020).

18. Teslovich, T. M. et al. Biological, clinical and population relevance of 95 loci for blood lipids. Nature 466, 707-713 (2010).

19. Adiels, M. et al. Overproduction of large VLDL particles is driven by increased liver fat content in man. Diabetologia 49, 755-765 (2006).

20. Diraison, F. \& Beylot, M. Role of human liver lipogenesis and reesterification in triglycerides secretion and in FFA reesterification. Am. J. Physiol. 274, E321-E327 (1998)

21. Fabbrini, E. et al. Alterations in adipose tissue and hepatic lipid kinetics in obese men and women with nonalcoholic fatty liver disease. Gastroenterology 134, 424-431 (2008).

22. Gibbons, G. F. Assembly and secretion of hepatic very-low-density lipoprotein. Biochem. J. 268, 1-13 (1990).

23. Salter, A. M., Wiggins, D., Sessions, V. A. \& Gibbons, G. F. The intracellular triacylglycerol/fatty acid cycle: a comparison of its activity in hepatocytes which secrete exclusively apolipoprotein (apo) B100 very-low-density lipoprotein (VLDL) and in those which secrete predominantly apoB48 VLDL. Biochem. J. 332, 667-672 (1998).

24. Dongiovanni, P. et al. Transmembrane 6 superfamily member 2 gene variant disentangles nonalcoholic steatohepatitis from cardiovascular disease. Hepatology 61, 506-514 (2015)

25. Stender, S. et al. Adiposity amplifies the genetic risk of fatty liver disease conferred by multiple loci. Nat. Genet. 49, 842-847 (2017).

26. Thangapandi, V. R. et al. Loss of hepatic Mboat7 leads to liver fibrosis. Gut 70, 940-950 (2021).

27. Baselli, G. A. et al. Liver transcriptomics highlights interleukin-32 as novel NAFLD-related cytokine and candidate biomarker. Gut 69, 1855-1866 (2020).

28. Kleiner, D. E. et al. Design and validation of a histological scoring system for nonalcoholic fatty liver disease. Hepatology 41, 1313-1321 (2005).

29. Abul-Husn, N. S. et al. A protein-truncating HSD17B13 variant and protection from chronic liver disease. N. Engl. J. Med. 378, 1096-1106 (2018).

30. Luukkonen, P. K. et al. MARC1 variant rs 2642438 increases hepatic phosphatidylcholines and decreases severity of non-alcoholic fatty liver disease in humans. J. Hepatol. 73, 725-726 (2020).

31. Jamialahmadi, O. et al. Exome-wide association study on alanine aminotransferase identifies sequence variants in the GPAM and APOE associated with fatty liver disease. Gastroenterology 160, 1634-1646.e1637 (2021).

32. Suvichapanich, S. et al. Genomewide association study confirming the association of NAT2 with susceptibility to antituberculosis drug-induced liver injury in thai patients. Antimicrob. Agents Chemother. 63, e02692-18 (2019).

33. D'Souza-Schorey, C. \& Chavrier, P. ARF proteins: roles in membrane traffic and beyond. Nat. Rev. Mol. Cell Biol. 7, 347-358 (2006)

34. Wang, Y. et al. Large scale identification of human hepatocellular carcinoma-associated antigens by autoantibodies. J. Immunol. $\mathbf{1 6 9}$ 1102-1109 (2002).

35. Dongiovanni, P. et al. Causal relationship of hepatic fat with liver damage and insulin resistance in nonalcoholic fatty liver. J. Intern. Med. 283, 356-370 (2018).

36. Tanaka, Y. et al. LPIAT1/MBOAT7 depletion increases triglyceride synthesis fueled by high phosphatidylinositol turnover. Gut 70, 180-193 (2021).

37. Victor, R. G. et al. The Dallas Heart Study: a population-based probability sample for the multidisciplinary study of ethnic differences in cardiovascular health. Am. J. Cardiol. 93, 1473-1480 (2004).

38. Szczepaniak, L. S. et al. Measurement of intracellular triglyceride stores by $\mathrm{H}$ spectroscopy: validation in vivo. Am. J. Physiol. 276, E977-E989 (1999).

39. Luukkonen, P. K. et al. Hepatic ceramides dissociate steatosis and insulin resistance in patients with non-alcoholic fatty liver disease. J. Hepatol. 64, 1167-1175 (2016).

40. Valenti, L. et al. The APOC3 T-455C and C-482T promoter region polymorphisms are not associated with the severity of liver damage independently of PNPLA3 I148M genotype in patients with nonalcoholic fatty liver. J. Hepatol. 55, 1409-1414 (2011).

41. Petta, S. et al. Glucokinase regulatory protein gene polymorphism affects liver fibrosis in non-alcoholic fatty liver disease. PLOS ONE 9, e87523 (2014).

42. Simonen, M. et al. Desmosterol in human nonalcoholic steatohepatitis. Hepatology 58, 976-982 (2013).
43. Canela-Xandri, O., Rawlik, K. \& Tenesa, A. An atlas of genetic associations in UK Biobank. Nat. Genet. 50, 1593-1599 (2018).

44. Crawford, K. et al. Medical consequences of pathogenic CNVs in adults: analysis of the UK Biobank. J. Med. Genet. 56, 131-138 (2019).

45. Linge, J. et al. Body composition profiling in the UK Biobank imaging study. Obesity 26, 1785-1795 (2018).

46. Linge, J., Whitcher, B., Borga, O. \& Dahlqvist Leinhard, O. Sub-phenotyping metabolic disorders using body composition: an individualized, nonparametric approach utilizing large data sets. Obesity (Silver Spring) 27, 1190-1199 (2019).

47. Bycroft, C. et al. The UK Biobank resource with deep phenotyping and genomic data. Nature 562, 203-209 (2018).

48. Sudlow, C. et al. UK Biobank: an open access resource for identifying the causes of a wide range of complex diseases of middle and old age. PLoS Med. 12, e1001779 (2015)

49. Prill, S. et al. The TM6SF2 E167K genetic variant induces lipid biosynthesis and reduces apolipoprotein B secretion in human hepatic 3D spheroids. Sci. Rep. 9, 11585 (2019).

50. Graham, M. J. et al. Antisense oligonucleotide inhibition of apolipoprotein C-III reduces plasma triglycerides in rodents, nonhuman primates, and humans. Circ. Res. 112, 1479-1490 (2013).

51. Patro, R., Duggal, G., Love, M. I., Irizarry, R. A. \& Kingsford, C. Salmon provides fast and bias-aware quantification of transcript expression. Nat. Methods 14, 417-419 (2017).

52. Pingitore, $\mathrm{P}$. et al. PNPLA3 overexpression results in reduction of proteins predisposing to fibrosis. Hum. Mol. Genet. 25, 5212-5222 (2016).

53. Pirazzi, C. et al. Patatin-like phospholipase domain-containing 3 (PNPLA3) I148M (rs738409) affects hepatic VLDL secretion in humans and in vitro. J. Hepatol. 57, 1276-1282 (2012).

54. Ölander, M. et al. A simple approach for restoration of differentiation and function in cryopreserved human hepatocytes. Arch. Toxicol. 93, 819-829 (2019).

55. Pingitore, P. et al. Human multilineage 3D spheroids as a model of liver steatosis and fibrosis. Int. J. Mol. Sci. 20, 1629 (2019).

56. Lindén, D., Sjöberg, A., Asp, L., Carlsson, L. \& Oscarsson, J. Direct effects of growth hormone on production and secretion of apolipoprotein B from rat hepatocytes. Am. J. Physiol. Endocrinol. Metab. 279, E1335-E1346 (2000).

57. Folch, J., Lees, M. \& Sloane Stanley, G. H. A simple method for the isolation and purification of total lipides from animal tissues. J. Biol. Chem. 226, 497-509 (1957)

58. Hansson, P. K., Asztély, A. K., Clapham, J. C. \& Schreyer, S. A. Glucose and fatty acid metabolism in McA-RH7777 hepatoma cells vs. rat primary hepatocytes: responsiveness to nutrient availability. Biochim. Biophys. Acta 1684, 54-62 (2004)

59. Østergaard, M. E. et al. Efficient synthesis and biological evaluation of 5 -GalNAc conjugated antisense oligonucleotides. Bioconjug. Chem. 26, 1451-1455 (2015).

60. Drescher, H. The influence of different fat sources on steatohepatitis and fibrosis development in the western diet mouse model of non-alcoholic steatohepatitis (NASH). Front. Physiol. 10, 770 (2019).

61. Carr, T. P., Andresen, C. J. \& Rudel, L. L. Enzymatic determination of triglyceride, free cholesterol, and total cholesterol in tissue lipid extracts. Clin. Biochem. 26, 39-42 (1993).

62. Balduzzi, S., Rücker, G. \& Schwarzer, G. How to perform a metaanalysis with R: a practical tutorial. Evid. Based Ment. Health 22, 153-160 (2019).

\section{Acknowledgements}

We thank Julia Kozlitina at University of Texas Southwestern Medical Center, Dallas, TX for the analysis of the DHS data. This work was supported by a project grant from the Swedish Research Council (Vetenskapsradet (VR), 2021-005208) (S.R.), the Swedish state under the Agreement between the Swedish government and the county councils (the ALF agreement, SU 2018-04276) (S.R.), the Swedish Diabetes Foundation (DIA2020-518) (S.R.), the Swedish Heart Lung Foundation (20200191) (S.R.), the Wallenberg Academy Fellows from the Knut and Alice Wallenberg Foundation (KAW 2017.0203) (S.R.), the Novonordisk Project grants in Endocrinology and Metabolism (NNF20OC0063883) (S.R.), AstraZeneca Agreement for Research, and Grant SSF ITM17-0384 (S.R.), Swedish Foundation for Strategic Research (S.R.), the MyFirst Grant AIRC n.16888, Ricerca Finalizzata Ministero della Salute RF-2016-02364358 (L.V.), Ricerca Corrente Fondazione IRCCS Ca' Granda Ospedale Maggiore Policlinico (L.V.) and the European Union (EU) Programme Horizon 2020 (under grant agreement no. 777377) for the project LITMUS-'Liver Investigation: Testing Marker Utility in Steatohepatitis' (L.V.)

\section{Author contributions}

R.M.M. performed in vitro studies, performed genetic analyses of the LBC and central European independent replication cohort, wrote the first draft of the manuscript and contributed to the study design, discussion and results interpretation. K.S. performed 
in vitro studies and wrote the first draft of the manuscript. A.L., Y.W., E.C., A.-C.A., G.P., S.K., M.P. and R.G.L. performed in vivo studies. C. Dix and M.C. generated the custom PSD3 polyclonal antibody. O.J. performed association analysis of the UK Biobank cohort. G.B. performed the transcriptomic analyses. J.P., P.D., H.Y.-J., S.P., P.K.L. and L.V. recruited patients from the $L B C$ and contributed to the discussion. L.V. also performed genotyping for the $\mathrm{LBC}$, designed the preliminary analysis of the $\mathrm{LBC}$, provided intellectual input into aspects of this study, and contributed to manuscript drafting and results interpretation. P.P., V.M., F.P., D.T., S.B., S.G., P.K.L., V.R. and C.X. contributed to the discussion and results interpretation. A.L.F. contributed to the discussion and results interpretation. F.S. and J.H. recruited patients from the replication cohort and contributed to the discussion. I.M. and M.M. performed the histopathology of human liver samples for PSD3, active ARF6 and provided their feedback for the manuscript. B.B. helped in primary hepatocyte cultures and read the manuscript and provided feedback. F.T. contributed to analysis and interpretation of data. V.K. and D.P. read the manuscript and provided their feedback. C.S. provided German liver samples. E.A. and C. Datz provided Austrian liver samples for the replication cohort. D.L. and S.R. conceived and planned the study, and contributed to the manuscript drafting, discussion and results interpretation. All authors contributed to drafting or revising the article and gave final approval of the version to be published.

\section{Competing interests}

A provisional patent application directed to the subject matter disclosed in this manuscript has been filed. A.L., A.-C.A., G.P., C. Dix, M.C. and D.L. are AstraZeneca employees. Y.W., S.K., M.P. and R.G.L. are Ionis Pharmaceuticals employees. S.R. has served as a consultant for AstraZeneca, Celgene, Sanofi, Amgen, Akcea Therapeutics, Camp4, Medacorp and Pfizer in the last 5 years. S.R. has received research grants from AstraZeneca, Sanofi and Amgen. L.V. reports having received speaking fees from MSD, Gilead, AlfaSigma, and AbbVie, having served as a consultant for Gilead, Pfizer, Astra Zeneca, Novo Nordisk, and having received research grants from Gilead. PKL was supported by the Novo Nordisk, Sigrid Jusélius and Instrumentarium Science Foundations. The remaining authors declare no competing interests.

\section{Additional information}

Supplementary information The online version contains supplementary material available at https://doi.org/10.1038/s42255-021-00518-0.

Correspondence and requests for materials should be addressed to Daniel Lindén or Stefano Romeo.

Peer review information Nature Metabolism thanks Anna Alisi, Herbert Tilg and the other, anonymous, reviewer for their contribution to the peer review of this work. Alfredo Gimenez-Cassina was the Primary Handling Editor.

Reprints and permissions information is available at www.nature.com/reprints.

Publisher's note Springer Nature remains neutral with regard to jurisdictional claims in published maps and institutional affiliations.

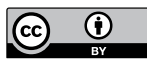

Open Access This article is licensed under a Creative Commons

Attribution 4.0 International License, which permits use, sharing, adaptation, distribution and reproduction in any medium or format, as long as you give appropriate credit to the original author(s) and the source, provide a link to the Creative Commons license, and indicate if changes were made. The images or other third party material in this article are included in the article's Creative Commons license, unless indicated otherwise in a credit line to the material. If material is not included in the article's Creative Commons license and your intended use is not permitted by statutory regulation or exceeds the permitted use, you will need to obtain permission directly from the copyright holder. To view a copy of this license, visit http://creativecommons. org/licenses/by/4.0/.

(C) The Author(s) 2022 


\section{Reporting Summary}

Nature Portfolio wishes to improve the reproducibility of the work that we publish. This form provides structure for consistency and transparency in reporting. For further information on Nature Portfolio policies, see our Editorial Policies and the Editorial Policy Checklist.

\section{Statistics}

For all statistical analyses, confirm that the following items are present in the figure legend, table legend, main text, or Methods section.

$\mathrm{n} / \mathrm{a}$ Confirmed

$\bigotimes$ The exact sample size $(n)$ for each experimental group/condition, given as a discrete number and unit of measurement

$\square$ A statement on whether measurements were taken from distinct samples or whether the same sample was measured repeatedly

The statistical test(s) used AND whether they are one- or two-sided

Only common tests should be described solely by name; describe more complex techniques in the Methods section.

$\square$ A description of all covariates tested

$\square$ \ A description of any assumptions or corrections, such as tests of normality and adjustment for multiple comparisons

$\square$ A full description of the statistical parameters including central tendency (e.g. means) or other basic estimates (e.g. regression coefficient)

AND variation (e.g. standard deviation) or associated estimates of uncertainty (e.g. confidence intervals)

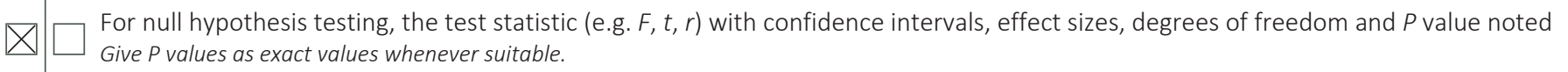

Х $\square$ For Bayesian analysis, information on the choice of priors and Markov chain Monte Carlo settings

Х $\square$ For hierarchical and complex designs, identification of the appropriate level for tests and full reporting of outcomes

\ $\square$ Estimates of effect sizes (e.g. Cohen's $d$, Pearson's $r$ ), indicating how they were calculated

Our web collection on statistics for biologists contains articles on many of the points above.

\section{Software and code}

Policy information about availability of computer code

Data collection No software was used for data collection. For UK Biobank, data collection was performed centrally.

Data analysis The following softwares have been used to analyze data or images: IBM SPSS statistics version 27, R version 3.6.1, MATLAB R2020b (academic license), and GraphPad Prism version 9, RSEM version 1.3.0, DESeq2 1.22.2 from Bioconductor version 3.8, DESeq2 R package 2_1.6.3, AxioVision 4.8 (Zeiss), Biopix iQ software version 2.3.1, Visiopharm Integrator System software (version 2018.09).

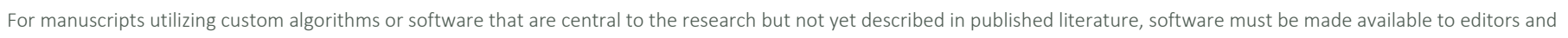

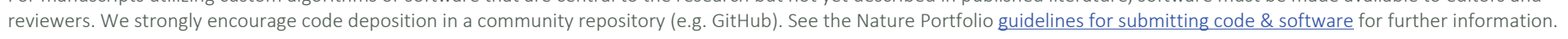

\section{Data}

Policy information about availability of data

All manuscripts must include a data availability statement. This statement should provide the following information, where applicable:

- Accession codes, unique identifiers, or web links for publicly available datasets

- A description of any restrictions on data availability

- For clinical datasets or third party data, please ensure that the statement adheres to our policy

All data associated with this study are present in the paper or the Supplementary Information. For UK Biobank, all individual-level phenotype/genotype data are accessible via a formal application to the UK Biobank http://www.ukbiobank.ac.uk. Antisense oligonucleotides associated with this study can be made available, upon reasonable request, to academic researchers under a material transfer agreement with AstraZeneca and lonis Pharmaceuticals. Due to study participants privacy data protection, RNA-seq data of the liver biopsies of the LBC can be made available only on request to the corresponding authors for collaborative projects. Bulk RNA seq data of the primary human hepatocytes are deposited in the NCBI SRA under the BioProject identifier PRJNA778044. All other data are available from 


\section{Field-specific reporting}

Please select the one below that is the best fit for your research. If you are not sure, read the appropriate sections before making your selection.

\Life sciences Behavioural \& social sciences Ecological, evolutionary \& environmental sciences

For a reference copy of the document with all sections, see nature.com/documents/nr-reporting-summary-flat.pdf

\section{Life sciences study design}

All studies must disclose on these points even when the disclosure is negative.

Sample size For genetic associations analyses, we performed the following power OR sample size calculations: in DHS considering 2,736 individuals and a MAF $>=0.1 \%$, we estimated to have a power $>80 \%$ to detect a $44 \%$ difference in liver fat content. To confirm our findings from the DHS in the LBC $(N=1,951)$, we selected only variants with a MAF in Europeans $>=5 \%$. Using this strategy, we estimated to have a power $>80 \%$ to detect $5 \%$ difference in the prevalence of liver steatosis. Based on our findings from the LBC, to be able to validate our results we estimated to need at least $\mathrm{N}=620$ individuals in the validation cohorts and we have $\mathrm{N}=674$ individuals in the "central European independent replication cohort", and N=6,994 from the "UK Biobank" replication cohort. Thus, we estimated to have enough power to validate our findings in these 2 cohorts. Statistical power or sample size calculations have been performed using IBM SPSS samplePower 3.

For preclinical NASH studies sample sizes were determined based on a combination of pilot studies using NASH-inducing diets and published literature indicating the smallest sample sizes required to achieve sufficiently low variability and statistical significance in liver fat, inflammation, and fibrosis endpoints and to achieve robust ASO-mediated target knockdown in C57BL/6 mice. Specifically, for NASH endpoints we used the following calculation to estimate sample sizes needed:

Sample size $=2 *\left(S D^{\wedge} 2\right)^{*}(1.96+0.842)^{\wedge} 2 / d^{\wedge} 2$ where $d=$ effect size (difference between mean values for control and experimental groups). (Reference: Charon and Kantharia, J Pharmacology \& Pharmaceuticals 2013).

Data exclusions

Exclusion criteria for all the study cohorts were pre-established as follows: for LBC and the "central European independent replication cohort", individuals with high alcohol intake (men, $>30 \mathrm{~g} /$ day; women, $>20 \mathrm{~g} /$ day), viral and autoimmune hepatitis or other causes of liver disease were excluded. Individuals with missing data on histological liver disease evaluation and/or PSD3 genotype were additionally excluded from the present study. For the UK Biobank, we restricted our analysis to the subset of unrelated white-British participants from the UK Biobank, after further removal of individuals with excessive relatives (more than 10 putative third-degree relatives), a mismatch between the self-reported and genetically inferred gender, putative sex chromosome aneuploidy, withdrawn consent, and those who were identified by the UK Biobank as outliers based on heterozygosity and missingness. For the DHS, for the present study, only individuals with measurement of hepatic triglyceride content have been included. Given the low prevalence of heavy drinking ( $>30 \mathrm{~g} /$ day) in this cohort, we did not exclude subjects based on alcohol intake. All analyses were based on cross-sectional data. No other exclusion criteria compared to the original study design.

Replication To confirm our findings from the DHS in the LBC ( $N=1,951)$, we selected only variants with a MAF in Europeans $>=5 \%$. Using this strategy, we estimated to have a power $>80 \%$ to detect $5 \%$ difference in the prevalence of liver steatosis. Based on our findings from the LBC, to be able to validate our results we estimated to need at least $\mathrm{N}=620$ individuals in the validation cohorts and we have $\mathrm{N}=674$ individuals in the "central European independent replication cohort", and N=6,994 from the "UK Biobank" replication cohort. Thus, we estimated to have enough power to validate our findings in these 2 cohorts. Statistical power or sample size calculations have been performed using IBM SPSS samplePower 3.

Randomization For in vivo studies, mice were randomized into study groups based on body weight and plasma ALT levels. For genetic studies, individuals have been allocated into the study groups based on the target genotype. Confounding factors (including, age, gender, BMI plus all the others relevant for each specific study) were controlled including these as covariates into the statical models. For in vitro studies, primary cells have been allocated into the study groups based on the target genotype; immortalized cells have been allocated into the study groups randomly.

Blinding

Histological evaluation of liver disease both in Human and in Murine samples has been performed by experienced histopathologists blinded from patients or study group condition and genotype.

\section{Reporting for specific materials, systems and methods}

We require information from authors about some types of materials, experimental systems and methods used in many studies. Here, indicate whether each material, system or method listed is relevant to your study. If you are not sure if a list item applies to your research, read the appropriate section before selecting a response. 


\begin{tabular}{l|l}
\hline n/a & Involved in the study \\
\hline & $\bigotimes$ Antibodies \\
$\square$ & $\bigotimes$ Eukaryotic cell lines \\
$\square$ & $\square$ Palaeontology and archaeology \\
$\square$ & $\bigotimes$ Animals and other organisms \\
$\square$ & $\square$ Clinical data \\
$\searrow$ & $\square$ Dual use research of concern
\end{tabular}

\section{Antibodies}

Antibodies used

Validation
Anti Calnexin (rabbit, for WB) (Sigma Aldrich C4731), Anti-Arf6 (mouse, for WB) provided from activation kit (Cell Biolabs Inc. STA-407-6), Anti-PSD3 (for WB) (Custom made), anti-active ARF6 (mouse, for IHC) (NewEast biosciences 26918), anti-PSD3(rabbit, for IHC) (ProSci 29-749), Anti-rabbit IgG HRP (for WB) (GE healthcare NA934V), Anti-Mouse IgG HRP(for WB) (GE healthcare NA931V), Goat Anti-Mouse Cat.\#170-6516 Bio-Rad Laboratories (secondary Ab for IHC), Goat Anti-Rabbit Cat. \#1706515 Bio-Rad Laboratories (secondary $\mathrm{Ab}$ for $\mathrm{IHC}$.

The PSD3 custom Ab was validated via silencing of endogenously expressed PSD3 in human hepatoma HepaRG cells (as described in the manuscript and in Supplementary figure 10).

For other antibodies, Species, application and any validation protocols/statements provided in the manufacturers website is stated below:

Anti Calnexin (rabbit) (Sigma Aldrich C4731)

Species: Rabbit, Tested applications: IP, Indirect immunofluorescence, microarray, western blot.

Species reactivity: canine, mouse, human, rat. Antibody validated by HeLa cells (human) extract separated on SDS-PAGE and probed with IgG fraction AB to calnexin (rabbit,cat no. C4731). The antibody was developed with Alk Phos APA Rabbit lgG(gt) Cat no, A9919 and a NBT/BCIP substrate.

species reactivity

Anti-Arf6 (mouse) provided from activation kit (Cell Biolabs Inc. STA-407-6)

The antibody reacts with Arf6 from human, bovine, canine, and rat. Antibody validated by western blot of HeLa cell lysate loaded with GDP and incubated with GGA3 PBD Agarose beads and HeLa cell lysate loaded with GTPYS and incubated with GGA3 PBD Agarose beads.

Anti-active ARF6 (mouse) (NewEast biosciences 26918)

Species: Mouse, Tested application: IP, IHC, Species reactivity: Anti-active Arf6 antibody recognizes active Arf6 from vertebrates. Antibody validated by Purified full-length Arf6 proteins immunoprecipitated after treated with GDP or GTPyS. Immunoprecipitation was done with the anti-active Arf6 monoclonal antibody (Cat. \# NEBA10223,Vita scientific). Immunoblot was with an anti-Arf6 polyclonal antibody (Cat. \#NEBA10020,Vita scientific).

Anti-PSD3(rabbit) (ProSci 29-749)

Species: Rabbit, Tested applications: ELISA, IHC, WB, Immunogen: Antibody produced in rabbits immunized with a synthetic peptide corresponding a region of human PSD3. Species reactivity: Human, mouse, rat. Antibody validated by human muscle on IHC and western blot on human A204 cells at $2.5 \mu \mathrm{g} / \mathrm{mL}$.

Amersham ECL Anti-rabbit IgG, Horseradish Peroxidase Linked Species-Specific Whole Antibody (from donkey) (GE healthcare NA934V)

Species: Donkey, Tested applications: Western blotting, ELISA, immunocytochemistry, Species reactivity: Rabbit. The antibody is prepared by hyper-immunizing donkeys with purified immunoglobulin fractions from normal rabbit serum to produce high affinity antibodies. Every batch is also QC tested in a Western blotting system. This is performed using Hybond ${ }^{\mathrm{TM}} E C \mathrm{~L}^{\mathrm{TM}}$ membrane containing serially diluted Beta-galactosidase protein and immundetected with primary antibody Anti-Beta-galactosidase and secondary antibody NA934, anti-rabbit HRP. Blots are detected using ECL and ECL Plus ${ }^{\text {TM }}$ detection systems.

Amersham ECL Anti-mouse IgG, peroxidase-linked whole antibody (from sheep) affinity purified general purpose reagent (GE healthcare NA931V)

Species: Sheep. Tested applications: western blotting. Species reactivity: Mouse.

The antibody is prepared by hyper-immunizing sheep with purified immunoglobulin fractions from normal mouse serum to produce high affinity antibodies. Every batch is also QC tested in a Western blotting system. This is performed using Hybond ${ }^{\mathrm{TM}} E C L^{\mathrm{TM}}$ membrane containing tubulin protein and immunodetected with: primary antibody, Monoclonal anti-tubulin; and secondary antibody NXA931, anti-mouse IgG, HRP GPR. Blots are detected using ECL and ECL Plus' ${ }^{\mathrm{TM}}$ detection systems

Goat anti Mouse IgG (H/L) polyclonal antibody (Cat no. 170-6516 Bio-Rad Laboratories)

Species: Goat, Tested applications: western blotting (Where this product has not been tested for use in a particular technique this does not necessarily exclude its use in such procedures). Species reactivity: mouse. Antiserum to mouse $\lg G(\mathrm{H} / \mathrm{L})$ was raised by repeated immunisation of goats with highly purified antigen. Antibody reacts with the heavy chains of mouse IgG, and the light chains common to the majority of mouse immunoglobulins. This antibody has not been cross adsorbed against other mouse immunoglobulins, and has been found to react substantially with antibody light chains common to all mouse antibodies. Therefore, significant cross reactivity to non-IgG primary antibodies may occur. Antibody validated by detection of recombinant Human CD33 with Mouse anti Human CD33 (MCA1271, Bio-Rad) in a Western blot analysis with Goat anti Mouse IgG:HRP (Cat no. 170-6516) at a 
Goat anti Rabbit IgG antibody (Cat no. 1706515 Bio-Rad Laboratories)

Species: goat, Tested applications; Immunohistology (frozen), ELISA, western blotting. Species reactivity: rabbit. Antisera to rabbit IgG were raised by repeated immunisations of goats with highly purified antigen. Purified IgG was prepared from whole serum by affinity chromatography.

\section{Eukaryotic cell lines}

Policy information about cell lines

Cell line source(s)

Authentication

Mycoplasma contamination

Commonly misidentified lines (See $\underline{\text { ICLAC }}$ register)
MCA RH-7777 cell line was purchased from ATCC; human hepatocytes Huh7 cell line was purchased from JCRB cell bank, Japan; Sf21 cells were purchased from Gibco( Cat no 11497013)

None of the cell lines used were authenticated.

All the cell lines used were tested negative for mycoplasma by providers

No commonly misidentified cell lines were used in the study.

\section{Animals and other organisms}

Policy information about studies involving animals; ARRIVE guidelines recommended for reporting animal research

Laboratory animals For in vivo studies six-week-old male C57BL/6 mice were used. All mice were obtained from the Jackson Laboratory (Bar Harbor, ME) and housed in cages on a 12 -h/12-h light/dark cycle at $22.5 \pm 2.5^{\circ} \mathrm{C}$ and $50 \pm 20 \%$ humidity, fed ad libitum and had free access to drinking water for the duration of the studies.

For custom PSD3 antibody generation three male, 22 weeks old New Zealand white rabbits were used.

Wild animals The study did not involve wild animals

Field-collected samples The study did not involve samples collected on the field

\begin{tabular}{l|l} 
Ethics oversight & The in vivo studies in mice were compliant with ethical regulation and approved by an institutional animal care and use committee
\end{tabular} and the IONIS local ethical committee, AAALAC Accreditation \#P-0305.

Note that full information on the approval of the study protocol must also be provided in the manuscript.

\section{Human research participants}

\section{Policy information about studies involving human research participants}

Population characteristics

Recruitment
For the DHS: $\mathrm{N}=2736$ individuals with mean age $46 \pm 10$ years, mean BMI $29 \pm 5 \mathrm{~kg} / \mathrm{m} 2$.and a total of 1533 individuals were female (56\%); 1328 (48\%) were African Americans, 882 (32\%) were European Americans and 465 (17\%) were Hispanics. For the LBC: a total of 1951 individuals with mean age $47 \pm 12$ year, mean BMI $37 \pm 9 \mathrm{~kg} / \mathrm{m} 2$, male gender $\mathrm{n}=909$ (47\%); of these individuals, $n=1,022(52 \%)$ were from University of Milan, Milan, Italy, $\mathrm{n}=374(19 \%)$ from the Palermo University Hospital, Palermo, Italy, $=410$ (21\%) from the Northern Savo Hospital District, Kuopio, Finland and $n=145(7 \%)$ from the Hospital District of Helsinki and Uusimaa, Finland.

Fore the Central European Cohort, a total of 674 individuals with mean age $45 \pm 12$ years, mean BMI $46 \pm 10 \mathrm{~kg} / \mathrm{m} 2$, male gender $n=236$ (35\%). Of these individuals, 559 (53\%) were from Germany, 83 (12\%) from Austria and 32 (5\%) from Switzerland.

For the UK Biobank: a total of 6,994 unrelated white British adult individuals were analyzed in the present study with mean age $55 \pm 7$ years, mean BMI $27 \pm 4 \mathrm{~kg} / \mathrm{m} 2$, male gender $n=3,392(48 \%)$.

The DHS is a multi-ethnic population-based sample of the Dallas County residents including individuals from three different ethnic groups, enrolled between 2000 and 2002. A probably sample of noninstitutionalized, English- or Spanish-speaking adult individuals aged $18-65$ years.

The primary basis for the sampling frame was the US Postal Service delivery sequence file. A probability sample with equal numbers of unrelated black and non-black women and men, was recruited. More details on the study design and recruitment can be found in the paper from Ronald $\mathrm{G}$ et al, the Am J of Cardiology (2004).

The LBC is a cross-sectional study of adult individuals of European descent who underwent liver biopsy for suspected nonalcoholic steatohepatitis (NASH) or severe obesity, consecutively enrolled in 3 independent European centres. To these, a total of 323 individuals from a fourth and independent Finnish centre has been included. Individuals with high alcohol intake 
(men, >30 g/day; women, >20 g/day), viral and autoimmune hepatitis or other causes of liver disease were excluded. More details on original study design and recruitment process can be found in the paper from Mancina RM et al., Gastroenterology (2016), Dongiovanni P et al., Hepatology (2015), and Luukkonen PK et al., J Hepatol 64, 1167-1175 (2016).

The UK Biobank is a large population-based prospective study comprising more than 500,000 adult individuals (aged between 40-69 years at recruitment) who visited 22 recruitment centres throughout the UK between 2006 and 2010 . For the overall study design and recruitment process please visit https://www.ukbiobank.ac.uk/media/gnkeyh2q/study-rationale.pdf .

For the present study, we restricted our analysis (cross-sectional) to a subset of unrelated white-British participants from the UK Biobank, after further removal of individuals with excessive relatives (more than 10 putative third-degree relatives), a mismatch between the self-reported and genetically inferred gender, putative sex chromosome aneuploidy, withdrawn consent, and those who were identified by the UK Biobank as outliers based on heterozygosity and missingness.

The central European cohort is a cross-sectional study of adult obese individuals with BMI $>30 \mathrm{~kg} / \mathrm{m} 2 \mathrm{who}$ underwent percutaneous or surgical liver biopsy. Individuals were consecutively recruited from tertiary referral centers in: Austria, Germany and Switzerland. Individuals with infectious (e.g. viral hepatitis, HIV), immunological, drug-induced hepatic steatosis (e.g. amiodarone, methotrexate, steroids, valproate, etc.) or hereditary causes (hereditary hemochromatosis, Wilson disease) of chronic liver disease were excluded by accepted measures. Individuals with self-reported average alcohol consumption > $30 \mathrm{~g} /$ day (in men) or $20 \mathrm{~g} /$ day (in women) were excluded.

Ethics oversight

The DHS was approved by the Institutional Review Board of University of Texas Southwestern Medical Centre. The LBC study was approved by the Ethics Committees of the Fondazione IRCCS Ca' Granda (Milan), Palermo University Hospital (Palermo), Northern Savo Hospital District in Kuopio (Finland), and the ethics committee of the Hospital District of Helsinki and Uusimaa (Finland).

The UK Biobank study received ethical approval from the National Research Ethics Service Committee North West MultiCentre Haydock (reference 16/NW/0274).

The Central European cohort was approved from local ethics committees of the participating centers.

All patients gave their written informed consent.

All studies were performed in accordance with the current version of the Helsinki Declaration.

Note that full information on the approval of the study protocol must also be provided in the manuscript. 